\title{
Adaptive Galerkin approximation algorithms for Kolmogorov equations in infinite dimensions
}

\author{
Christoph Schwab · Endre Süli
}

Received: 23 July 2012 / Published online: 7 March 2013

(C) Springer Science+Business Media New York 2013

\begin{abstract}
Space-time variational formulations and adaptive Wiener-Hermite polynomial chaos Galerkin discretizations of Kolmogorov equations in infinite dimensions, such as Fokker-Planck and Ornstein-Uhlenbeck equations for functions defined on an infinite-dimensional separable Hilbert space $H$, are developed. The wellposedness of these equations in the Hilbert space $\mathrm{L}^{2}(H, \mu)$ of functions on the infinite-dimensional domain $H$, which are square-integrable with respect to a Gaussian measure $\mu$ with trace class covariance operator $Q$ on $H$, is proved. Specifically, for the infinite-dimensional Fokker-Planck equation, adaptive space-time Galerkin discretizations, based on a wavelet polynomial chaos Riesz basis obtained by tensorization of biorthogonal piecewise polynomial wavelet bases in time with a spatial Wiener-Hermite polynomial chaos arising from the Wiener-Itô decomposition of $\mathrm{L}^{2}(H, \mu)$, are introduced. The resulting space-time adaptive Wiener-Hermite polynomial Galerkin discretization algorithms of the infinite-dimensional PDE are proved to converge quasioptimally in the sense that they produce sequences of finite-dimensional approximations that attain the best possible convergence rates afforded by best $N$-term approximations of the solution from tensor-products of multiresolution (wavelet) time-discretizations and the Wiener-Hermite polynomial chaos in $\mathrm{L}^{2}(H, \mu)$. As a consequence, the proposed adaptive Galerkin solution algorithms exhibit dimension-independent performance, which is optimal with respect to the algebraic best $N$-term rate afforded by the solution and the polynomial degree and regularity
\end{abstract}

C. Schwab $(\varangle)$

Seminar for Applied Mathematics (SAM), ETH Zurich, HG G57.1,

CH 8092 Zurich, Switzerland

e-mail: schwab@math.ethz.ch

E. Süli

Mathematical Institute, University of Oxford, 24-29 St Giles, Oxford OX1 3LB, UK

e-mail: suli@maths.ox.ac.uk 
of the multiresolution (wavelet) time-discretizations in the finite-dimensional case, in particular. All constants in our error and complexity bounds are shown to be independent of the number of "active" coordinates identified by the proposed adaptive Galerkin approximation algorithms. The computational work and memory required by the proposed algorithms scale linearly with the support size of the coefficient vectors that arise in the approximations, with dimension-independent constants.

\section{Introduction}

Partial differential equations in infinite dimensions arise in a number of relevant applications; most notably as forward and backward Kolmogorov equations for stochastic Partial Differential Equations (SPDEs for short), we refer to, e.g., [5,4] and the references therein. Citing [10, p. X], "parabolic equations on Hilbert spaces appear in mathematical physics to model systems with infinitely many degrees of freedom. Typical examples are provided by spin configurations in statistical mechanics and by crystals in solid state theory. Infinite-dimensional parabolic equations provide an analytic description of infinite-dimensional diffusion processes in such branches of applied mathematics as population biology, fluid dynamics, and mathematical finance." In spite of their prominence in a range of relevant applications, the numerical solution of PDEs in infinite dimensions appears to have received only scant attention in the mathematical literature. Numerical approximations to such equations are mostly attempted by path simulations in the corresponding stochastic partial differential equation. Their path-wise solutions belong to function spaces over finite-dimensional domains and can be, therefore, approximated by standard discretization techniques, combined with Monte Carlo path sampling. In the present paper, we propose and analyze a novel, deterministic, adaptive, spectral-Galerkin approach to the construction of finite-dimensional numerical approximations to the deterministic forward Kolmogorov (or, Fokker-Planck) equation in infinite-dimensional spaces, which exhibit certain optimality properties. The proposed approach is based on space-time variational formulations of these equations, which are posed in Gel'fand-triples of Sobolev spaces over a separable Hilbert space $H$, a typical example being $H=\mathbb{R}^{\mathbb{N}}$ ) with respect to a Gaussian measure $\mu$, and on the use of Riesz bases of these spaces, which have been developed for linear and nonlinear parabolic PDEs set in spaces of functions defined on finite-dimensional domains in $[20,6,15]$. In this paper, we present a class of adaptive Galerkin discretizations of Fokker-Planck equations for functions defined on an infinite-dimensional separable Hilbert space $H$ that are square-integrable with respect to a Gaussian measure $\mu$ on $H$ with respect to a given covariance operator $Q$ on $H$, which we assume to be of trace-class. In contrast with the considerations in $[7,8,20]$, we use a spectral Galerkin method on $H$ here, based on Wiener-Hermite polynomial chaos expansions in terms of a sequence of independent Gaussian random variables on $H$, and, as in [20], a wavelet type Riesz basis with respect to the time variable. We establish stability and optimality of the adaptive space-time Galerkin discretizations thus obtained. Specifically, we use here the general approach developed by Cohen, Dahmen and DeVore in a series of papers (cf. [7,8] and the references therein) to show the optimality of nonlinear, adaptive Galerkin approximations of elliptic operator equations on bounded domains in $\mathbb{R}^{d}$. These tools were extended in 
[20] to parabolic evolution equations, using multiresolution (wavelet) bases, again on bounded domains in $\mathbb{R}^{d}$. In the present paper we prove, for a particular class of secondorder differential operators acting on spaces of functions on the infinite-dimensional domain $H$, which typically arise as forward equations for SPDEs, numerical sparsity of the bi-infinite wavelet polynomial chaos (WPC) matrix representation of the parabolic operator.

Our adaptive Galerkin discretization is shown to generate, in particular, a family of stable finite-dimensional discretizations of the Fokker-Planck equation on the infinite-dimensional domain $H$, which are quasioptimal finite-dimensional truncations of the parabolic evolution problem on the infinite-dimensional domain. The use of a spectral basis of Wiener-Hermite polynomial chaos type obviates "meshing" the infinite-dimensional "domain" $H$ of solutions of the parabolic equation.

The structure of this paper is as follows: in the next section, we first present two space-time variational formulations of abstract, linear parabolic equations set in a Gel'fand evolution triple $\mathcal{V} \subset \mathcal{H} \simeq \mathcal{H}^{*} \subset \mathcal{V}^{*}$. We then prepare the corresponding formulation of an infinite-dimensional Fokker-Planck equation by recapitulating basic facts about Gaussian measures on separable Hilbert spaces. In Section 4, we prove well-posedness of the second of our two space-time variational formulations in the infinite-dimensional pivot space $\mathcal{H}=\mathrm{L}^{2}(H, \mu)$, where $H$ denotes an infinite-dimensional separable Hilbert space and $\mu$ is a Gaussian measure on $H$ with a trace-class covariance operator $Q$. We shall establish, in particular, that the solution operator of the variational formulation is an isomorphism between suitable solution and data spaces. We then focus on the formulation and the analysis of space-time-adaptive Galerkin approximations. We outline the general principle in Section 5, where we introduce the idea of conversion of abstract, well-posed operator equations on separable Hilbert spaces to equivalent, bi-infinite matrix-vector problems in the sequence space $\ell^{2}(\mathbb{N})$. After reviewing $N$-term approximations in $\ell^{2}(\mathbb{N})$, we introduce abstract adaptive Galerkin approximation algorithms that construct sequences of $N$-term approximations, which, while not being best $N$-term approximations, are optimal in the sense that these approximations converge asymptotically at the rate afforded by the best $N$-term approximation, provided that certain conditions are met by the operators and the Riesz bases used to discretize them. In Section 8 the abstract concepts are specialized to infinite-dimensional Kolmogorov equations. Notably, a Wiener-Hermite polynomial chaos type Riesz basis in $\mathrm{L}^{2}(H, \mu)$ and a wavelet basis in time is used as the basis for the Galerkin discretization. In Section 9 we verify the abstract assumptions for the specific equations of interest, and in Section 10 we consider the more general setting of nonsymmetric equations with drift, leading to our main result concerning optimality of adaptive Galerkin discretizations with dimension-independent bounds, in Section 11.

\section{Space-time variational formulation of abstract parabolic problems}

\subsection{Abstract parabolic equations}

Given $T>0$ and a Gel'fand triple 


$$
\mathcal{V} \hookrightarrow \mathcal{H} \cong \mathcal{H}^{*} \hookrightarrow \mathcal{V}^{*}
$$

for separable Hilbert spaces $\mathcal{H}$ and $\mathcal{V}$ over the field $\mathbb{R}$ of real numbers, with the continuous and dense embeddings signified by the symbol $\hookrightarrow$, we consider the abstract parabolic differential equation

$$
\partial_{t} u+A u=f \text { in } \mathcal{V}^{*} \text { for } t \in(0, T),
$$

where $A \in \mathcal{L}\left(\mathcal{V}, \mathcal{V}^{*}\right)$, with $\|A\|_{\mathcal{L}\left(\mathcal{V}, \mathcal{V}^{*}\right)}=M_{a}>0$, satisfies $A=A^{*}$, and we suppose that the following Garding inequality holds:

$$
\exists m_{a}>0 \quad \exists \kappa \geq 0 \quad \forall v \in \mathcal{V}: \quad \mathfrak{a}(v, v):=\mathcal{V} *\langle A v, v\rangle_{\mathcal{V}} \geq m_{a}\|v\|_{\mathcal{V}}^{2}-\kappa\|v\|_{\mathcal{H}}^{2}
$$

Here $\mathcal{V} *|\cdot, \cdot\rangle_{\mathcal{V}}$ denotes the duality pairing between $\mathcal{V}^{*}$, the dual space of $\mathcal{V}$, and the space $\mathcal{V}$. As our aim is to develop the numerical analysis of space-time adaptive Galerkin discretizations of infinite-dimensional parabolic problems, we begin, following [20], by presenting weak formulations that are amenable to adaptive Galerkin discretizations. To this end, we consider the Bochner spaces $\mathrm{L}^{2}(0, T ; \mathcal{V}), \mathrm{L}^{2}(0, T ; \mathcal{H})$ and $\mathrm{L}^{2}\left(0, T ; \mathcal{V}^{*}\right)$ and we define

$$
\mathrm{H}^{1}(0, T ; \mathcal{V}):=\left\{u \in \mathrm{L}^{2}(0, T ; \mathcal{V}): u^{\prime} \in \mathrm{L}^{2}(0, T ; \mathcal{V})\right\}
$$

as well as

$$
\begin{aligned}
& \mathrm{H}_{0,\{0\}}^{1}(0, T ; \mathcal{V}):=\left\{u \in \mathrm{H}^{1}(0, T ; \mathcal{V}): u(0)=0 \text { in } \mathcal{V}\right\} \\
& \mathrm{H}_{0,\{T\}}^{1}(0, T ; \mathcal{V}):=\left\{u \in \mathrm{H}^{1}(0, T ; \mathcal{V}): u(T)=0 \text { in } \mathcal{V}\right\}
\end{aligned}
$$

Here and throughout the rest of the paper $u^{\prime}$ will signify $\mathrm{d} u / \mathrm{d} t$ or $\partial u / \partial t$, depending on the context.

In the variational formulation of the parabolic problem, an important role is played by the space $\mathcal{X}$ defined by

$$
\mathcal{X}:=\mathrm{L}^{2}(0, T ; \mathcal{V}) \cap \mathrm{H}^{1}\left(0, T ; \mathcal{V}^{*}\right),
$$

which we equip with the norm $\|\cdot\| \mathcal{X}$ defined by

$$
\|v\| \mathcal{X}:=\left(\|v\|_{\mathrm{L}^{2}(0, T ; \mathcal{V})}^{2}+\left\|v^{\prime}\right\|_{\mathrm{L}^{2}\left(0, T ; \mathcal{V}^{*}\right)}^{2}\right)^{\frac{1}{2}}
$$

With $\mathcal{V}, \mathcal{H}$ and $\mathcal{V}^{*}$ as in the triple (2.1) the following continuous embedding holds:

$$
\mathcal{X} \hookrightarrow \mathrm{C}([0, T] ; \mathcal{H})
$$

(in the sense that any $v \in \mathcal{X}$ is equal almost everywhere to a function that is uniformly continuous as a mapping from the nonempty compact interval $[0, T]$ of the real line 
into $\mathcal{H})$. Therefore, for $u \in \mathcal{X}$ and $t \in[0, T]$, the values $u(t)$ are well-defined in $\mathcal{H}$ and there exists a constant $C=C(T)>0$ such that

$$
\forall u \in \mathcal{X} \quad \forall t \in[0, T]: \quad\|u(t)\|_{\mathcal{H}} \leq C\|u\|_{\mathcal{X}}
$$

In particular, for $u \in \mathcal{X}$ the values $u(0)$ and $u(T)$ are well-defined in $\mathcal{H}$ and

$$
\mathcal{X}_{0,\{0\}}:=\{u \in \mathcal{X}: u(0)=0 \text { in } \mathcal{H}\}, \quad \mathcal{X}_{0,\{T\}}:=\{u \in \mathcal{X}: u(T)=0 \text { in } \mathcal{H}\}
$$

are closed linear subspaces of $\mathcal{X}$. Henceforth we shall write $\mathcal{Y}=\mathrm{L}^{2}(0, T ; \mathcal{V})$ and we denote by $\mathcal{Y}^{*}$ the dual space of $\mathcal{Y}$, which is isomorphic to $\mathrm{L}^{2}\left(0, T ; \mathcal{V}^{*}\right)$ identifying $\mathrm{L}^{2}(0, T ; \mathcal{H})$ with its own dual.

\subsection{First space-time variational formulation}

We consider (2.2) in the special case when

$$
L u:=\partial_{t} u+A u=f \in \mathcal{Y}^{*} \text { in }(0, T), \quad u(0)=u_{0} \in \mathcal{H},
$$

in conjunction with a suitable homogenous boundary condition incorporated in the (domain of) definition of the linear operator $A$. We begin by considering the case when the initial condition is also homogeneous, i.e., $u_{0}=0 \in \mathcal{H}$.

The first space-time variational formulation of the parabolic problem (2.8) is based on the bilinear form

$$
(u, v) \in \mathcal{X} \times \mathcal{Y} \mapsto \mathfrak{B}(u, v):=\int_{0}^{T}\left(\mathcal{V} *\left\langle u^{\prime}, v\right\rangle \mathcal{V}+\mathfrak{a}(u, v)\right) \mathrm{d} t \in \mathbb{R}
$$

Then, the space-time weak formulation of the parabolic problem (2.8) with homogeneous initial condition $u_{0}=0$ in $\mathcal{H}$ reads as follows: given $f \in \mathcal{Y}^{*}$, find $u \in \mathcal{X}_{0,\{0\}}$ such that

$$
\mathfrak{B}(u, v)=f(v) \quad \forall v \in \mathcal{Y} .
$$

\subsection{Second space-time variational formulation}

In (2.10), the initial condition $u(0)=0$ was incorporated in the definition of the function space $\mathcal{X}_{0,\{0\}}$ in which the solution to the problem was sought. To accommodate a nonhomogeneous initial condition, one may proceed in (at least) two different ways. If

$$
u(0)=u_{0} \neq 0 \text { in } \mathcal{H}
$$

then one can for example first subtract from $u$ a function $u_{p} \in \mathcal{X}$ such that $\left.u_{p}\right|_{t=0}=u_{0}$; we may, in particular, choose for this purpose

$$
u_{p}=\mathrm{e}^{-t} u_{0}, \quad \text { for } u_{0} \in \mathcal{V} \subset \mathcal{H} \text {. }
$$


The disadvantage of this approach is that $u_{0} \in \mathcal{V}$ is needed (instead of $u_{0} \in \mathcal{H}$ ), which can be viewed as an unnecessarily restrictive demand on the regularity of the initial datum $u_{0}$.

Alternatively, in order to relax the regularity requirement $u_{0} \in \mathcal{V}$ to $u_{0} \in \mathcal{H}$, one may impose (2.11) weakly, either by enforcing it via a multiplier as in [20] or by a space-time variational formulation, which incorporates it as a natural boundary condition as follows: in (2.10) we integrate the time derivative by parts using that

$$
\begin{array}{r}
\int_{0}^{T} \mathcal{V} *\left\langle u^{\prime}, v\right\rangle_{\mathcal{V}} \mathrm{d} t= \\
u, \int_{0}^{T} \mathcal{V} *\left\langle v^{\prime}, u\right\rangle \mathcal{V} \mathrm{d} t+\left.(u, v)\right|_{0} ^{T}, \\
u, v \mathrm{~L}^{2}(0, T ; \mathcal{V}) \cap \mathrm{H}^{1}\left(0, T ; \mathcal{V}^{*}\right)
\end{array}
$$

If $u(0)=u_{0} \neq 0$ in $\mathcal{H}$, we require that

$$
v(T)=0
$$

Thus, (2.13) and (2.14) lead to the weak formulation (2.17) below. To state it, we recall the spaces $\mathcal{Y}=\mathrm{L}^{2}(0, T ; \mathcal{V})$ and

$$
\mathcal{X}=\mathrm{L}^{2}(0, T ; \mathcal{V}) \cap \mathrm{H}^{1}\left(0, T ; \mathcal{V}^{*}\right)
$$

and the subspaces (cf. also (2.5))

$\mathcal{X}_{0,\{0\}}:=\{u \in \mathcal{X}: u(0)=0$ in $\mathcal{H}\}, \quad \mathcal{X}_{0,\{T\}}:=\{u \in \mathcal{X}: u(T)=0$ in $\mathcal{H}\}$,

equipped with the norm of $\mathcal{X}$; we shall write $\|\cdot\|_{\mathcal{X}_{0,\{0\}}}$ and $\|\cdot\| \mathcal{X}_{0,\{T\}}$ to indicate that the norm of $\mathcal{X}$ is applied to an element of $\mathcal{X}_{0,\{0\}}$ and $\mathcal{X}_{0,\{T\}}$, respectively. Thanks to the continuous embedding (2.7), $\mathcal{X}_{0,\{0\}}$ and $\mathcal{X}_{0,\{T\}}$ are closed, linear subspaces of $\mathcal{X}$; in particular the expression (2.13) is meaningful for $u, v \in \mathcal{X}$. The variational form of the parabolic problem (2.8) with weak enforcement of the initial condition, which we shall also refer to as the space-time adjoint weak formulation, then reads: given $u_{0} \in \mathcal{H}$ and $f \in \mathcal{X}_{0,\{T\}}^{*}$, find

$$
u \in \mathcal{Y}: \quad \mathfrak{B}^{*}(u, v)=\ell^{*}(v) \quad \forall v \in \mathcal{X}_{0,\{T\}}
$$

Here the bilinear form $\mathfrak{B}^{*}(\cdot, \cdot)$ is given by

$$
\mathfrak{B}^{*}(u, v):=\int_{0}^{T}\left(-\mathcal{V}^{*}\left\langle v^{\prime}, u\right\rangle \mathcal{V}+\mathfrak{a}(u, v)\right) \mathrm{d} t
$$

and the linear functional $\ell^{*}$ is defined by

$$
\ell^{*}(v):=\mathcal{X}^{*}\langle f, v\rangle_{\mathcal{X}}+\left(u_{0}, v(0)\right)_{\mathcal{H}}
$$

For future reference we collect some simple properties of the functional $\ell^{*}$ in a proposition. 
Proposition 2.1 For $f \in \mathcal{X}_{0,\{T\}}^{*} \simeq \mathrm{L}^{2}\left(I ; \mathcal{V}^{*}\right)+\left(\mathrm{H}_{0,\{T\}}^{1}\right)^{*}(I ; \mathcal{V}) \simeq \mathrm{L}^{2}(I) \otimes \mathcal{V}^{*}$ $+\left(\mathrm{H}_{0,\{T\}}^{1}(I)\right)^{*} \otimes \mathcal{V}$ and, for any $u_{0} \in \mathcal{H}$, the functional $\ell^{*}$ in (2.19) is linear and continuous on $\mathcal{X}_{0,\{T\}}$, i.e., there exists a constant $C>0$ such that

$$
\forall v \in \mathcal{X}_{0,\{T\}}: \quad\left|\ell^{*}(v)\right| \leq C\left(\|f\|_{\mathcal{X}_{0,\{T\}}^{*}}+\left\|u_{0}\right\|_{\mathcal{H}}\right)\|v\|_{\mathcal{X}_{0,\{T\}}} .
$$

With all preparatory considerations in place, we are now ready to discuss the question of well-posedness of the variational formulations (2.10) and (2.17).

\subsection{Well-posedness}

By proceeding analogously as in the proof of [20, Theorem 5.1], we arrive at the following result.

Theorem 2.2 Suppose that $\mathcal{V}$ and $\mathcal{H}$ are separable Hilbert spaces over the field $\mathbb{R}$ such that $\mathcal{V} \hookrightarrow \mathcal{H} \simeq \mathcal{H}^{*} \hookrightarrow \mathcal{V}^{*}$ with continuous and dense embeddings. Assume further that the bilinear form $\mathfrak{a}(\cdot, \cdot): \mathcal{V} \times \mathcal{V} \rightarrow \mathbb{R}$ is continuous, i.e.,

$$
\exists M_{a}>0 \quad \forall w, v \in \mathcal{V}: \quad|\mathfrak{a}(w, v)| \leq M_{a}\|v\| \mathcal{V}\|w\| \mathcal{V},
$$

and coercive in the sense that it satisfies a Garding inequality, i.e., there exist $m_{a}>0$ and $\kappa \geq 0$ such that

$$
\forall v \in \mathcal{V}: \quad \mathfrak{a}(v, v) \geq m_{a}\|v\|_{\mathcal{V}}^{2}-\kappa\|v\|_{\mathcal{H}}^{2} .
$$

Then, there exists a positive constant $\gamma=\gamma\left(m_{a}, M_{a}, \kappa, T\right)$ such that the bilinear form $\mathfrak{B}(\cdot, \cdot)$, as defined in (2.9), satisfies the following inequalities, referred to as the inf-sup condition:

$$
\inf _{u \in \mathcal{X} \mathcal{X}_{0,\{0\} \backslash\{0\}}} \sup _{v \in \mathcal{Y} \backslash\{0\}} \frac{\mathfrak{B}(u, v)}{\|u\| \mathcal{X}\|v\| \mathcal{Y}} \geq \gamma>0
$$

and

$$
\forall v \in \mathcal{Y} \backslash\{0\}: \sup _{u \in \mathcal{X}_{0,\{0\}}} \mathfrak{B}(u, v)>0
$$

Furthermore, there exists a positive constant $\gamma=\gamma\left(m_{a}, M_{a}, \kappa, T\right)$, such that $\mathfrak{B}^{*}(\cdot, \cdot)$ in $(2.18)$ satisfies the following inf-sup condition:

$$
\inf _{u \in \mathcal{Y} \backslash\{0\}} \sup _{v \in \mathcal{X}_{0,\{T\} \backslash\{0\}}} \frac{\mathfrak{B}^{*}(u, v)}{\|u\| \mathcal{Y}\|v\| \mathcal{X}_{0,\{T\}}} \geq \gamma
$$

and

$$
\forall v \in \mathcal{X}_{0,\{T\}} \backslash\{0\}: \quad \sup _{u \in \mathcal{Y}} \mathfrak{B}^{*}(u, v)>0 .
$$


Moreover, the bilinear forms $\mathfrak{B}(\cdot, \cdot)$ and $\mathfrak{B}^{*}(\cdot, \cdot)$ defined in (2.9) and in (2.17), respectively, induce boundedly invertible linear operators $B \in \mathcal{L}\left(\mathcal{X}_{0,\{0\}}, \mathcal{Y}^{*}\right)$ and $B^{*} \in \mathcal{L}\left(\mathcal{Y},\left(\mathcal{X}_{0,\{T\}}\right)^{*}\right)$, where the spaces $\mathcal{X}_{0,\{0\}}, \mathcal{X}_{0,\{T\}}$ and $\mathcal{Y}$ are defined in $(2.15)$, (2.16).

The stability conditions satisfied by $\mathfrak{B}^{*}(\cdot, \cdot)$ imply the following result concerning unique solvability of the corresponding variational formulation (2.17). An analogous result holds for (2.10), thanks to the stability properties of $\mathfrak{B}(\cdot, \cdot)$.

Theorem 2.3 For every $u_{0} \in \mathcal{H}$ and every $f \in \mathcal{X}_{0,\{T\}}^{*}=\mathrm{L}^{2}\left(I ; \mathcal{V}^{*}\right)+\mathrm{H}_{0,\{T\}}^{-1}(I ; \mathcal{V})$, there exists a unique weak solution $u \in \mathcal{Y}=\mathrm{L}^{2}(0, T ; \mathcal{V})$ of $(2.8)$ in the sense that $u$ satisfies (2.17). Moreover, the operator $L: \mathcal{Y} \rightarrow \mathcal{X}_{0,\{T\}}^{*}$ is an isomorphism.

The proof of this theorem is analogous to that of [20, Theorem 5.1], and is therefore omitted. We are now ready to consider a concrete realization of this abstract theoretical framework, which concerns parabolic PDEs in infinite dimensions.

\section{Gaussian measures}

Suppose that $H$ is a separable Hilbert space with norm $\|\cdot\|_{H}$ and inner product $(\cdot, \cdot)_{H}$ over the field of real numbers. We denote by $\mathrm{L}(H)$ the space of all bounded linear operators from $H$ into $H$, equipped with the associated operator norm $\|\cdot\|_{\mathrm{L}(H)}$, and we denote by $\mathrm{L}^{+}(H)$ the subset of $H$ consisting of all nonnegative, symmetric and bounded, linear operators on $H$. Finally, $\mathfrak{B}(H)$ will signify the $\sigma$-algebra of all Borel subsets of $H$. We recall that a bounded linear operator $R \in \mathrm{L}(H)$ is said to be trace-class if there exist two sequences $\left(a_{k}\right)_{k=1}^{\infty}$ and $\left(b_{k}\right)_{k=1}^{\infty}$ in $H$ such that

$$
R h=\sum_{k=1}^{\infty}\left(h, a_{k}\right)_{H} b_{k}, \quad h \in H,
$$

and

$$
\sum_{k=1}^{\infty}\left\|a_{k}\right\|_{H}\left\|b_{k}\right\|_{H}<\infty .
$$

The set of all elements of $\mathrm{L}(H)$ that are trace-class will be denoted by $\mathrm{L}_{1}(H)$. We note that if $R \in \mathrm{L}_{1}(H)$, then $R$ is a compact linear operator on $H$. The set $\mathrm{L}_{1}(H)$, endowed with the usual operations of addition and scalar multiplication, is a Banach space with the norm

$$
\begin{array}{r}
\|R\|_{\mathrm{L}_{1}(H)}:=\inf \left\{\sum_{k=1}^{\infty}\left\|a_{k}\right\|_{H}\left\|b_{k}\right\|_{H}: R h=\sum_{k=1}^{\infty}\left(h, a_{k}\right)_{H} b_{k},\right. \\
\left.h \in H, \quad\left(a_{k}\right)_{k=1}^{\infty},\left(b_{k}\right)_{k=1}^{\infty} \subset H\right\} .
\end{array}
$$


Assuming that $R \in \mathrm{L}_{1}(H)$, the trace $\operatorname{Tr} R$ of $R$ is defined by the formula

$$
\operatorname{Tr} R=\sum_{k=1}^{\infty}\left(R e_{k}, e_{k}\right)_{H}
$$

where at this stage $\left(e_{k}\right)_{k=1}^{\infty}$ is any complete orthonormal basis in $H$. In particular if $R \in \mathrm{L}_{1}(H)$ is expressed by (3.1), then

$$
\operatorname{Tr} R=\sum_{k=1}^{\infty}\left(a_{k}, b_{k}\right)_{H}
$$

The definition of trace being independent of the choice of the basis, we have that $|\operatorname{Tr} R| \leq\|R\|_{\mathrm{L}_{1}(H)}$; we refer to Pietsch [18]) for further details on such operators. Let $\mathbb{R}^{\mathbb{N}}$ denote linear space of all sequences $x=\left(x_{k}\right)_{k=1}^{\infty}$ of real numbers, equipped with the metric

$$
\varrho(x, y):=\sum_{k=1}^{\infty} 2^{-k} \frac{\left|x_{k}-y_{k}\right|}{1+\left|x_{k}-y_{k}\right|}, \quad x, y \in \mathbb{R}^{\mathbb{N}} .
$$

Let further $\ell^{2}(\mathbb{N})$ denote the Hilbert space of all sequences $x=\left(x_{k}\right)_{k=1}^{\infty} \in \mathbb{R}^{\mathbb{N}}$ such that

$$
\|x\|_{\ell^{2}(\mathbb{N})}:=\left(\sum_{k=1}^{\infty}\left|x_{k}\right|^{2}\right)^{1 / 2}<+\infty
$$

the inner product on $\ell^{2}(\mathbb{N})$ is defined by $(x, y)_{\ell^{2}(\mathbb{N})}:=\sum_{k=1}^{\infty} x_{k} y_{k}$, for $x, y \in \ell^{2}(\mathbb{N})$.

Let us further consider, for $a \in \mathbb{R}$ and for $\lambda>0$, the Gaussian measure on $\mathbb{R}$ with mean $a$ and standard deviation $\lambda$ defined by

$$
N_{a, \lambda}(\mathrm{d} x):=\frac{1}{\sqrt{2 \pi \lambda}} \mathrm{e}^{-\frac{(x-a)^{2}}{2 \lambda}} \mathrm{d} x .
$$

For $x \in H$, we shall then write $x_{k}=\left(x, e_{k}\right)_{H}, k \in \mathbb{N}$. The following result is stated as Theorem 1.2.1 in [10].

Theorem 3.1 Suppose that $a \in H$ and $Q \in \mathrm{L}_{1}^{+}(H)$. Then, there exists a complete orthonormal system $\left(e_{k}\right)_{k=1}^{\infty}$ in $H$ and a sequence $\left(\lambda_{k}\right)_{k=1}^{\infty}$ of positive real numbers, the eigenvalues of $Q$, such that $Q e_{k}=\lambda_{k} e_{k}, k \in \mathbb{N}$, with the eigenvalues $\lambda_{k}$ assumed to be enumerated in decreasing order (and repeated according to their multiplicity), and accumulating only at zero.

Moreover, there exists a unique probability measure $\mu$ on $(H, \mathfrak{B}(H))$ such that

$$
\int_{H} \mathrm{e}^{l(h, x)_{H}} \mu(\mathrm{d} x)=\mathrm{e}^{l(a, h)_{H}} \mathrm{e}^{-\frac{1}{2}(Q h, h)_{H}}, \quad h \in H,
$$


and $\mu$ is the restriction to $H$ (identified with the Hilbert space $\ell^{2}(\mathbb{N})$ ) of the product measure

$$
\bigotimes_{k=1}^{\infty} \mu_{k}=\bigotimes_{k=1}^{\infty} N_{a_{k}, \lambda_{k}}
$$

defined on $\left(\mathbb{R}^{\mathbb{N}}, \mathfrak{B}\left(\mathbb{R}^{\mathbb{N}}\right)\right)$, with $a_{k}=\left(a, e_{k}\right)_{H}$.

We shall write $\mu=N_{a, Q}$, and we call $a$ the mean and the trace-class operator $Q$ the covariance operator of $\mu$. The measure $\mu$ will be referred to as a Gaussian measure on $H$ with mean $a$ and covariance operator $Q$. If the law of a random variable is a Gaussian measure, then the random variable is said to be Gaussian. In particular, Theorem 3.1 implies that a random variable $X$ with values in $H$ is Gaussian if, and only if, for any $h \in H$ the real-valued random variable $(h, X)_{H}$ is Gaussian.

For $\mu=N_{a, Q}$, we denote by $\mathrm{L}^{2}(H, \mu)$ the Hilbert space of all square-integrable (equivalence classes of) functions from $H$ into $\mathbb{R}$ with inner product

$$
(u, v)_{\mathrm{L}^{2}(H, \mu)}=\int_{H} u(x) v(x) \mu(\mathrm{d} x), \quad u, v \in \mathrm{L}^{2}(H, \mu)
$$

and norm

$$
\|u\|_{\mathrm{L}^{2}(H, \mu)}=(u, u)_{\mathrm{L}^{2}(H, \mu)}^{\frac{1}{2}}, \quad u \in \mathrm{L}^{2}(H, \mu) .
$$

Analogously, we shall denote by $\mathrm{L}^{2}(H, \mu ; H)$ the Hilbert space of all squareintegrable (equivalence classes of) functions from $H$ into $H$ with inner product

$$
(u, v)_{\mathrm{L}^{2}(H, \mu ; H)}=\int_{H}(u(x), v(x))_{H} \mu(\mathrm{d} x), \quad u, v \in \mathrm{L}^{2}(H, \mu ; H)
$$

and norm

$$
\|u\|_{\mathrm{L}^{2}(H, \mu ; H)}=(u, u)_{\mathrm{L}^{2}(H, \mu ; H)}^{\frac{1}{2}}, \quad u \in \mathrm{L}^{2}(H, \mu ; H) .
$$

Throughout the rest of the paper, $\mu=N_{Q}:=N_{0, Q}$, where $Q \in \mathrm{L}_{1}^{+}(H)$. Moreover, to avoid having to repeatedly distinguish cases in the ensuing analysis, we shall assume that $\operatorname{Ker}(Q)=\{0\}$ unless explicitly stated otherwise. We note, however, that all results obtained in the following remain valid with obvious modifications when the measure $\mu$ is supported on a finite-dimensional Hilbert space $H$, in which case the covariance operator $Q$ is of finite rank.

The subspace $Q^{1 / 2}(H)=\left\{Q^{1 / 2} h: h \in H\right\}$ is called the reproducing kernel of the Gaussian measure $N_{Q}:=N_{0, Q}$. If $\operatorname{Ker}(Q)=\{0\}$ as has been assumed, then $Q^{1 / 2}(H)$ is dense in $H$. In fact, if $x_{0} \in H$ is such that $\left(Q^{1 / 2} h, x_{0}\right)_{H}=0$ for all $h \in H$, then $Q^{1 / 2} x_{0}=0$, and therefore $Q x_{0}=0$, which implies that $x_{0}=0$. 
For $Q \in \mathrm{L}_{1}^{+}(H)$ such that $\operatorname{Ker}(Q)=\{0\}$, we introduce the isomorphism $W$ from $H$ into $\mathrm{L}^{2}\left(H, N_{Q}\right)$ as follows: for $f \in Q^{1 / 2}(H)$ let $W_{f} \in \mathrm{L}^{2}\left(H ; N_{Q}\right)$ be defined by

$$
W_{f}(x)=\left(Q^{-1 / 2} f, x\right)_{H}, \quad x \in H .
$$

Let $\mu=N_{Q}$. We define Hermite polynomials in $\mathrm{L}^{2}(H, \mu)$. Let us consider to this end the set $\Gamma$ of all mappings $\gamma: n \in \mathbb{N} \rightarrow \gamma_{n} \in\{0\} \cup \mathbb{N}$, such that $|\gamma|:=$ $\sum_{k=1}^{\infty} \gamma_{k}<+\infty$. Clearly $\gamma \in \Gamma$ if, and only if, $\gamma_{n}=0$ for all, except possibly finitely many, $n \in \mathbb{N}$. For any $\gamma \in \Gamma$ we define the Hermite polynomial

$$
H_{\gamma}(x):=\prod_{k \geq 1} H_{\gamma_{k}}\left(W_{e_{k}}(x)\right), \quad x \in H,
$$

where the factors in the product on the right-hand side are defined by

$$
H_{n}(\xi)=\frac{(-1)^{n}}{\sqrt{n !}} \mathrm{e}^{\frac{\xi^{2}}{2}} \frac{\mathrm{d}^{n}}{\mathrm{~d} \xi^{n}}\left(\mathrm{e}^{-\frac{\xi^{2}}{2}}\right), \quad \xi \in \mathbb{R}, \quad n \in\{0\} \cup \mathbb{N} .
$$

Note that $H_{0} \equiv 1$, so that for each $\gamma \in \Gamma$ the countable product $H_{\gamma}(x)$ contains only finitely many nontrivial factors and is, therefore, well-defined. Moreover, with the Gaussian measure $\mu=N_{Q}$ being a countable product measure, we have that

$$
\forall \gamma, \gamma^{\prime} \in \Gamma: \quad\left(H_{\gamma}, H_{\gamma^{\prime}}\right)_{\mathrm{L}^{2}(H, \mu)}=\delta_{\gamma, \gamma^{\prime}} .
$$

We shall denote by $E(H)$ the linear space spanned by all exponential functions, that is all functions $\varphi: x \in H \mapsto \varphi(x) \in \mathbb{R}$ of the form

$$
\varphi(x)=\mathrm{e}^{(h, x)_{H}}, \quad h \in H .
$$

It follows from Proposition 1.2.5 in Da Prato and Zabczyk [10] that $E(H)$ is dense in $\mathrm{L}^{2}(H, \mu)$. On account of the separability of $H, \mathrm{~L}^{2}(H, \mu)$ is separable.

For any $k \in \mathbb{N}$ we consider the partial derivative in the direction $e_{k}$ (with $e_{k}$ as above), defined by

$$
\mathrm{D}_{k} \varphi(x)=\lim _{\varepsilon \rightarrow 0} \frac{1}{\varepsilon}\left(\varphi\left(x+\varepsilon e_{k}\right)-\varphi(x)\right), \quad x \in H, \varphi \in E(H) .
$$

Thus, if $\varphi(x)=\mathrm{e}^{(h, x) H}$ with $h \in H$, then clearly

$$
\mathrm{D}_{k} \varphi(x)=\mathrm{e}^{(h, x)_{H}} h_{k}, \quad \text { where } h_{k}=\left(h, e_{k}\right)_{H} .
$$

On $E(H)$, we define the linear operator

$$
\mathrm{D}: E(H) \subset \mathrm{L}^{2}(H, \mu) \rightarrow \mathrm{L}^{2}(H, \mu ; H) \quad \text { by } \mathrm{D} \varphi(x):=\sum_{k=1}^{\infty} \mathrm{D}_{k} \varphi(x) e_{k}, \quad x \in H .
$$


Thanks to Proposition 9.2.2 in Da Prato and Zabczyk [10], $\mathrm{D}_{k}$ is a closable linear operator for all $k \in \mathbb{N}$. If $\varphi$ belongs to the domain of the closure of $\mathrm{D}_{k}$, which we shall still denote by $\mathrm{D}_{k}$, we shall say that $\mathrm{D}_{k} \varphi$ belongs to $\mathrm{L}^{2}(H, \mu)$.

Analogously, by Proposition 9.2.4 in Da Prato and Zabczyk [10], D is a closable linear operator. If $\varphi$ belongs to the domain of the closure of $\mathrm{D}$, which we shall still denote by $\mathrm{D}$, we shall say that $\mathrm{D} \varphi$ belongs to $\mathrm{L}^{2}(H, \mu ; H)$.

For $\mu=N_{Q}$, let us denote by $\mathrm{W}^{1,2}(H, \mu)$ the linear space of all functions $\varphi$ $\in \mathrm{L}^{2}(H, \mu)$ such that $\mathrm{D} \varphi \in \mathrm{L}^{2}(H, \mu ; H)$, equipped with the inner product

$$
(\varphi, \psi)_{\mathrm{W}^{1,2}(H, \mu)}:=(\varphi, \psi)_{\mathrm{L}^{2}(H, \mu)}+\int_{H}(\mathrm{D} \varphi(x), \mathrm{D} \psi(x))_{H} \mu(\mathrm{d} x)
$$

and norm $\|\varphi\|_{\mathrm{W}^{1,2}(H, \mu)}=(\varphi, \varphi)_{\mathrm{W}^{1,2}(H, \mu)}^{\frac{1}{2}}$. Then, the Sobolev space $\mathrm{W}^{1,2}(H, \mu)$ is complete, and is therefore a separable Hilbert space.

For any $\varphi \in \mathrm{L}^{2}(H, \mu)$, we have that

$$
\varphi=\sum_{\gamma \in \Gamma} \varphi_{\gamma} H_{\gamma}, \quad \text { where } \varphi_{\gamma}:=\left(\varphi, H_{\gamma}\right)_{\mathrm{L}^{2}(H, \mu)}, \quad \text { and } \quad\|\varphi\|_{\mathrm{L}^{2}(H, \mu)}^{2}=\sum_{\gamma \in \Gamma}\left|\varphi_{\gamma}\right|^{2} \text {. }
$$

The next theorem, proved independently by Da Prato, Malliavin and Nualart [9] and Peszat [17] provides an analogous characterization of functions belonging to $\mathrm{W}^{1,2}(H, \mu)$ in terms of the complete orthonormal basis $\left(H_{\gamma}\right)_{\gamma \in \Gamma}$.

Theorem 3.2 A function $\varphi \in \mathrm{L}^{2}(H, \mu)$ belongs to $\mathrm{W}^{1,2}(H, \mu)$ if, and only if,

$$
\sum_{\gamma \in \Gamma \backslash\{0\}}\left\langle\gamma, \lambda^{-1}\right\rangle\left|\varphi_{\gamma}\right|^{2}<+\infty
$$

where $\varphi_{\gamma}:=\left(\varphi, H_{\gamma}\right)_{\mathrm{L}^{2}(H, \mu)},\left\langle\gamma, \lambda^{-1}\right\rangle:=\sum_{k=1}^{\infty} \gamma_{k} \lambda_{k}^{-1}$, and $\left(\lambda_{k}\right)_{k=1}^{\infty}$ is the sequence of (positive) eigenvalues (repeated according to their multiplicity) of the covariance operator $Q \in \mathrm{L}_{1}^{+}(H), \operatorname{Ker}(Q)=\{0\}$. Moreover, if (3.6) holds, then

$$
\|\varphi\|_{\mathrm{W}^{1,2}(H, \mu)}^{2}=\|\varphi\|_{\mathrm{L}^{2}(H, \mu)}^{2}+\sum_{\gamma \in \Gamma \backslash\{0\}}\left\langle\gamma, \lambda^{-1}\right\rangle\left|\varphi_{\gamma}\right|^{2}
$$

Identifying $\mathrm{L}^{2}(H, \mu)$ with its own dual $\mathrm{L}^{2}(H, \mu)^{*}$, we obtain

$$
\varphi \in\left(\mathrm{W}^{1,2}(H, \mu)\right)^{*} \Longleftrightarrow \sum_{\gamma \in \Gamma \backslash\{0\}}\left(\left\langle\gamma, \lambda^{-1}\right\rangle\right)^{-1}\left|\varphi_{\gamma}\right|^{2}<+\infty
$$

Finally, the embedding of $\mathrm{W}^{1,2}(H, \mu)$ into $\mathrm{L}^{2}(H, \mu)$ is compact.

For a proof of these statements we refer, for example, to [10, Theorem 9.2.12]. As $E(H)$ is dense in both $\mathrm{L}^{2}(H, \mu)$ and $\mathrm{W}^{1,2}(H, \mu)$, the embedding of $\mathrm{W}^{1,2}(H, \mu)$ into $\mathrm{L}^{2}(H, \mu)$ is also dense. 


\section{Fokker-Planck equation in countably many dimensions}

For $k \in \mathbb{N}$, let us denote by $D_{k}$ the set $\mathbb{R}^{d}$ equipped with the Gaussian measure

$$
\begin{aligned}
\mu_{k}\left(\mathrm{~d} q_{k}\right)= & N_{a_{k}, \Sigma_{k}}\left(\mathrm{~d} q_{k}\right):=\frac{1}{(2 \pi)^{d / 2}\left[\operatorname{det}\left(\Sigma_{k}\right)\right]^{1 / 2}} \\
& \times \exp \left(-\frac{1}{2}\left(q_{k}-a_{k}\right)^{\top} \Sigma_{k}^{-1}\left(q_{k}-a_{k}\right)\right) \mathrm{d} q_{k}
\end{aligned}
$$

with mean $a_{k} \in \mathbb{R}^{d}$ and positive definite covariance matrix $\Sigma_{k} \in \mathbb{R}^{d \times d}$. We shall assume henceforth that $a_{k}=0$ for all $k \in \mathbb{N}$ and that the covariance operator $Q$, represented by the infinite block-diagonal matrix $\Sigma:=\operatorname{diag}\left(\Sigma_{1}, \Sigma_{2}, \ldots\right)$, with $d \times d$ diagonal blocks $\Sigma_{k}, k=1,2, \ldots$, is trace-class. We define the infinite-dimensional configuration domain

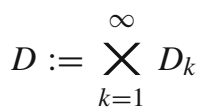

so that

$$
q:=\left(q_{1}^{\top}, q_{2}^{\top}, \ldots\right)^{\top} \in D, \quad q_{k} \in D_{k}, \quad k=1,2, \ldots
$$

We equip the infinite-dimensional domain $D$ with the product measure

$$
\mu:=\bigotimes_{k=1}^{\infty} \mu_{k}=\bigotimes_{k=1}^{\infty} N_{0, \Sigma_{k}}
$$

Let $\mathbf{A}=\left(A_{i j}\right)_{i, j=1}^{\infty} \in \mathbb{R}^{\mathbb{N} \times \mathbb{N}}$ be a symmetric bi-infinite matrix, i.e., $A_{i j}=A_{j i}$ for all $i, j \in \mathbb{N}$. Suppose further that there exists a real number $\gamma_{0}>0$ such that

$$
\sum_{i, j=1}^{\infty} A_{i j} \xi_{i} \xi_{j} \geq \gamma_{0}\|\xi\|_{\ell^{2}}^{2} \text { for all } \xi=\left(\xi_{i}\right)_{i=1}^{\infty} \in \ell^{2}(\mathbb{N})
$$

and a real number $\gamma_{1}>0$ such that for all $\xi=\left(\xi_{i}\right)_{i=1}^{\infty} \in \ell^{2}(\mathbb{N})$ and $\eta=\left(\eta_{i}\right)_{i=1}^{\infty} \in$ $\ell^{2}(\mathbb{N})$.

$$
\left|\sum_{i, j=1}^{\infty} A_{i j} \xi_{i} \eta_{j}\right| \leq \gamma_{1}\|\xi\|_{\ell^{2}}\|\eta\|_{\ell^{2}}
$$

Example 4.1 As an example of a bi-infinite matrix A that satisfies (4.1) and (4.2), we mention

$$
\mathbf{A}[\epsilon]:=\operatorname{tridiag}\left\{\left(\epsilon_{j}, 1, \epsilon_{j}\right): j=1,2, \ldots\right\},
$$

where the sequence $\epsilon=\left\{\epsilon_{j}\right\}_{j \geq 1}$ is assumed to be such that $\|\epsilon\|_{\infty}<1 / 2$. Then, the matrix $\mathbf{A}[\epsilon]$ in (4.3) satisfies (4.1) with $\gamma_{0}=1-2\|\epsilon\|_{\infty}$ and (4.2) with $\gamma_{1}=1+2\|\epsilon\|_{\infty}$. 
Example 4.2 An important application of our methods are Fokker-Planck equations with state spaces of finite, but high dimension. Such equations arise, among others, in connection with mesoscale descriptions of polymeric fluids. We refer to [1-3] and the references therein. Here, our results apply if we consider $\mathbf{A}$ of block-diagonal from $\mathbf{A}=\operatorname{diag}\left\{\mathbf{A}_{11}, \mathbf{A}_{22}\right\}$, where $\mathbf{A}_{11} \in \mathbb{R}^{d \times d}$ is symmetric, positive definite, and $\mathbf{A}_{22}$ is as in Example 4.1. Then, (4.1) and (4.2) hold with constants $0<\gamma_{0} \leq \gamma_{1}<\infty$ depending on the spectrum of $\mathbf{A}_{11}$ and on $d$, however.

We now choose $\mathcal{H}:=\mathrm{L}^{2}(D ; \mu)$ and $\mathcal{V}:=\mathrm{W}^{1,2}(D, \mu)$. Thanks to Theorem 3.2 and the subsequent discussion, $\mathcal{V} \hookrightarrow \mathcal{H}$, with continuous, dense and compact embedding. In order to state the space-time variational formulation of the infinite-dimensional Fokker-Planck equation under consideration here, we again write $\mathcal{Y}=\mathrm{L}^{2}(0, T ; \mathcal{V})$ and, guided by the abstract framework in Sections 2.2, 2.3 we consider the function space

$$
\mathcal{X}=\mathrm{L}^{2}(0, T ; \mathcal{V}) \cap \mathrm{H}^{1}\left(0, T ; \mathcal{V}^{*}\right)
$$

and its subspaces $\mathcal{X}_{0,\{0\}}, \mathcal{X}_{0,\{T\}}$ as in (2.16), which are norm-closed subspaces of $\mathcal{X}$ when equipped with the norm of $\mathcal{X}$.

With these spaces, the space-time adjoint weak formulation of the infinitedimensional Fokker-Planck equation reads as follows: given $\widehat{\psi}_{0} \in \mathcal{H}$ and $g \in \mathcal{X}_{0,\{T\}}^{*}$, find

$$
\widehat{\psi} \in \mathcal{Y}: \quad \mathfrak{B}^{*}(\widehat{\psi}, \widehat{\varphi})=\ell^{*}(\widehat{\varphi}) \quad \forall \widehat{\varphi} \in \mathcal{X}_{0,\{T\}},
$$

where the bilinear form $\mathfrak{B}^{*}(\cdot, \cdot): \mathcal{Y} \times \mathcal{X}_{0,\{T\}} \rightarrow \mathbb{R}$ is defined by

$$
\mathfrak{B}^{*}(\widehat{\psi}, \widehat{\varphi}):=\int_{0}^{T}\left(-\mathcal{V}^{*}\left\langle\widehat{\varphi}^{\prime}, \widehat{\psi}\right\rangle_{\mathcal{V}}+\mathfrak{a}(\widehat{\psi}, \widehat{\varphi})\right) \mathrm{d} t
$$

with

$$
\mathfrak{a}(\widehat{\psi}, \widehat{\varphi})=\sum_{i, j=1}^{\infty} A_{i j}\left(\nabla_{q_{i}} \widehat{\psi}, \nabla_{q_{j}} \widehat{\varphi}\right)_{\left[\mathrm{L}^{2}(D, \mu)\right]^{d}}
$$

where $\nabla_{q_{i}}$ denotes the gradient with respect to the co-ordinates $q_{i} \in D_{k} \subset \mathbb{R}^{d}$ and the linear functional $\ell^{*}$ is defined by

$$
\ell^{*}(\widehat{\varphi}):=\left(\widehat{\psi}_{0}, \widehat{\varphi}(0)\right)_{\mathrm{L}^{2}(D, \mu)}+\int_{0}^{T} \mathcal{V}^{*}\langle g(t), \hat{\varphi}\rangle_{\mathcal{V}} \mathrm{d} t
$$

The well-posedness of the infinite-dimensional Fokker-Planck equation is now an immediate consequence of Theorems 2.2 and 2.3.

Theorem 4.3 For the bilinear form $\mathfrak{B}^{*}(\cdot, \cdot)$ in (4.6) there exists a constant $\gamma>0$ such that 


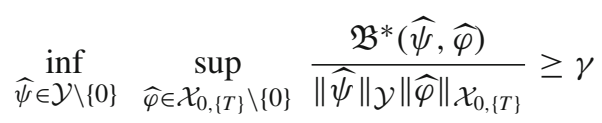

and

$$
\forall \widehat{\varphi} \in \mathcal{X}_{0,\{T\}} \backslash\{0\}: \quad \sup _{\widehat{\psi} \in \mathcal{Y}} \mathfrak{B}^{*}(\widehat{\psi}, \widehat{\varphi})>0 .
$$

Furthermore, for each $\widehat{\psi}_{0} \in \mathcal{H}$ and each $g \in \mathcal{X}_{0,\{T\}}^{*}$, there exists a unique $\widehat{\psi} \in \mathcal{Y}$ $=\mathrm{L}^{2}(0, T ; \mathcal{V})$ that satisfies $(4.5) ;$ and the linear operator $B^{*} \in \mathcal{L}\left(\mathcal{Y}, \mathcal{X}_{0,\{T\}}^{*}\right)$ induced by $\mathfrak{B}^{*}(\cdot, \cdot)$ is an isomorphism.

Following [20], we shall next develop a class of adaptive Galerkin discretization algorithms for (4.5), along the lines of adaptive wavelet discretizations of boundedly invertible operator equations considered in $[7,8]$. These algorithms exhibit, in particular, "stability by adaptivity", i.e., their stability follows directly from the stability (4.9), (4.10) of the continuous, infinite-dimensional problem through suitable Riesz bases of the spaces $\mathcal{Y}$ and $\mathcal{X}_{0,\{T\}}$, which we shall construct. Notably, in the present context, these algorithms are dimensionally robust by design: as we shall prove, they deliver a sequence of approximate solutions with finitely supported coefficient vectors, i.e., with only finitely many variables $q_{k}$ being 'active' in each iteration. We will establish certain optimality properties for these finitely supported Galerkin solutions, with all constants in the error and complexity bounds being absolute, i.e., independent of the number of active variables. We first develop the necessary concepts in an abstract setting, before applying them to the Fokker-Planck equation (4.5) in space-time variational form.

\section{Well-posed operator equations as bi-infinite matrix problems}

Let us denote for a moment by $\mathcal{X}$ and $\mathcal{Y}$ two generic separable Hilbert spaces over the field $\mathbb{R}$ of real numbers, and let us assume that we have available a Riesz basis $\Psi^{\mathcal{X}}=\left\{\psi_{\lambda}^{\mathcal{X}}: \lambda \in \nabla_{\mathcal{X}}\right\}$ for $\mathcal{X}$, meaning that the synthesis operator

$$
s_{\Psi^{\mathcal{X}}}: \ell^{2}\left(\nabla_{\mathcal{X}}\right) \rightarrow \mathcal{X}: \mathbf{c} \mapsto \mathbf{c}^{\top} \Psi^{\mathcal{X}}:=\sum_{\lambda \in \nabla_{\mathcal{X}}} c_{\lambda} \psi_{\lambda}^{\mathcal{X}}
$$

is boundedly invertible. By identifying $\ell^{2}\left(\nabla_{\mathcal{X}}\right)$ with its dual, the adjoint of $s_{\Psi} \mathcal{X}$, known as the analysis operator, is

$$
s_{\Psi^{\mathcal{X}}}^{*}: \mathcal{X}^{*} \rightarrow \ell^{2}\left(\nabla_{\mathcal{X}}\right): g \mapsto\left[g\left(\psi_{\lambda}^{\mathcal{X}}\right)\right]_{\lambda \in \nabla_{\mathcal{X}}}
$$

Similarly, let $\Psi^{\mathcal{Y}}=\left\{\psi_{\lambda}^{\mathcal{Y}}: \lambda \in \nabla \mathcal{Y}\right\}$ be a Riesz basis for $\mathcal{Y}$, with synthesis operator $s_{\Psi} \mathcal{Y}$ and its adjoint $s_{\Psi}^{*} \mathcal{Y}$. 
Now let $B \in \mathcal{L}\left(\mathcal{X}, \mathcal{Y}^{*}\right)$ be boundedly invertible. Then also its adjoint $B^{*}$ $\in \mathcal{L}\left(\mathcal{Y}, \mathcal{X}^{*}\right)$ is boundedly invertible and, for every $f \in \mathcal{Y}^{*}, f^{*} \in \mathcal{X}^{*}$, the operator equations

$$
B u=f, \quad B^{*} u^{*}=f^{*}
$$

admit unique solutions $u \in \mathcal{X}, u^{*} \in \mathcal{Y}$. Writing $u=s_{\Psi^{\mathcal{X}}} \mathbf{u}$ and $u^{*}=s_{\Psi} \mathcal{Y} \mathbf{u}^{*}$, these operator equations are equivalent to the bi-infinite matrix-vector problems

$$
\mathbf{B u}=\mathbf{f}, \quad \mathbf{B}^{*} \mathbf{u}^{*}=\mathbf{f}^{*},
$$

where the "load vectors" $\mathbf{f}=s_{\Psi^{\mathcal{Y}}}^{*} f=\left[f\left(\psi_{\lambda}^{\mathcal{Y}}\right)\right]_{\lambda \in \nabla_{\mathcal{Y}}} \in \ell^{2}\left(\nabla_{\mathcal{Y}}\right), \mathbf{f}^{*}=s_{\Psi^{\mathcal{X}}}^{*}$ $f=\left[f\left(\psi_{\lambda}^{\mathcal{X}}\right)\right]_{\lambda \in \nabla_{\mathcal{X}}} \in \ell^{2}\left(\nabla_{\mathcal{X}}\right)$; and the "stiffness" or system matrices $\mathbf{B}$ and $\mathbf{B}^{*}$ given by

$$
\mathbf{B}=s_{\Psi}^{*} \mathcal{Y} B s_{\Psi^{\mathcal{X}}}=\left[\left(B \psi_{\mu}^{\mathcal{X}}\right)\left(\psi_{\lambda}^{\mathcal{Y}}\right)\right]_{\lambda \in \nabla_{\mathcal{Y}}, \mu \in \nabla_{\mathcal{X}}} \in \mathcal{L}\left(\ell^{2}\left(\nabla_{\mathcal{X}}\right), \ell^{2}\left(\nabla_{\mathcal{Y}}\right)\right)
$$

and with $\mathbf{B}^{*}$ defined analogously, are boundedly invertible. We consider the associated bilinear forms

$$
\begin{aligned}
\mathfrak{B}(\cdot, \cdot): \mathcal{X} \times \mathcal{Y} \rightarrow \mathbb{R}:(w, v) \mapsto(B w)(v), \quad \mathfrak{B}^{*}(\cdot, \cdot): \mathcal{Y} \times \mathcal{X} & \rightarrow \mathbb{R}:(v, w) \\
& \mapsto\left(B^{*} v\right)(w),
\end{aligned}
$$

and introduce the notations

$$
\mathbf{B}=\mathfrak{B}\left(\Psi^{\mathcal{X}}, \Psi^{\mathcal{Y}}\right), \quad \mathbf{f}=f\left(\Psi^{\mathcal{Y}}\right), \quad \mathbf{B}^{*}=\mathfrak{B}^{*}\left(\Psi^{\mathcal{Y}}, \Psi^{\mathcal{X}}\right), \quad \mathbf{f}^{*}=f^{*}\left(\Psi^{\mathcal{X}}\right)
$$

With the Riesz constants

$$
\begin{aligned}
& \Lambda_{\Psi^{\mathcal{X}}}^{\mathcal{X}}:=\left\|s_{\Psi^{\mathcal{X}}}\right\|_{\ell^{2}\left(\nabla_{\mathcal{X}}\right) \rightarrow \mathcal{X}}=\sup _{0 \neq \mathbf{c} \in \ell^{2}\left(\nabla_{\mathcal{X}}\right)} \frac{\left\|\mathbf{c}^{\top} \Psi^{\mathcal{X}}\right\|_{\mathcal{X}}}{\|\mathbf{c}\|_{\ell^{2}\left(\nabla_{\mathcal{X}}\right)}}, \\
& \lambda_{\Psi^{\mathcal{X}}}^{\mathcal{X}}:=\left\|s_{\Psi^{\mathcal{X}}}^{-1}\right\|_{\mathcal{X} \rightarrow \ell^{2}\left(\nabla_{\mathcal{X}}\right)}^{-1}=\inf _{0 \neq \mathbf{c} \in \ell^{2}\left(\nabla_{\mathcal{X}}\right)} \frac{\left\|\mathbf{c}^{\top} \Psi^{\mathcal{X}}\right\|_{\mathcal{X}}}{\| \mathbf{c}_{\ell^{2}\left(\nabla_{\mathcal{X}}\right)}},
\end{aligned}
$$

and $\Lambda_{\Psi}^{\mathcal{Y}}$ and $\lambda_{\Psi^{\mathcal{Y}}}^{\mathcal{Y}}$ defined analogously, we obviously have that

$$
\begin{aligned}
\|\mathbf{B}\|_{\ell^{2}\left(\nabla_{\mathcal{X}}\right) \rightarrow \ell^{2}\left(\nabla_{\mathcal{Y}}\right)} & \leq\|B\|_{\mathcal{X} \rightarrow \mathcal{Y} *} \Lambda_{\Psi_{\mathcal{X}}^{\mathcal{X}}}^{\mathcal{X}} \Lambda_{\Psi^{\mathcal{Y}}}^{\mathcal{Y}}, \\
\left\|\mathbf{B}^{-1}\right\|_{\ell^{2}(\nabla \mathcal{Y}) \rightarrow \ell^{2}\left(\nabla_{\mathcal{X}}\right)} & \leq \frac{\left\|B^{-1}\right\|_{\mathcal{Y}} \rightarrow \mathcal{X}}{\lambda_{\Psi_{\mathcal{X}}^{\mathcal{X}} \lambda_{\Psi}^{\mathcal{Y}}}^{\mathcal{Y}}} .
\end{aligned}
$$




\section{Best $N$-term approximations and approximation classes}

We say that $\mathbf{u}_{N} \in \ell^{2}\left(\nabla_{\mathcal{X}}\right)$ is a best $N$-term approximation of $\mathbf{u} \in \ell^{2}\left(\nabla_{\mathcal{X}}\right)$, if it is the best possible approximation to $u$ in the norm of $\ell^{2}\left(\nabla_{\mathcal{X}}\right)$, given a budget of $N$ coefficients. Determining the best $N$-term approximation $\mathbf{u}_{N}$ of $\mathbf{u}$ requires searching the infinite vector $\mathbf{u}$, which is not feasible, i.e., actually locating $\mathbf{u}_{N}$ may not be practically feasible. Nevertheless, rates of convergence afforded by best $N$-term approximations serve as a benchmark for concrete numerical schemes. To this end, we collect all $\mathbf{u}$ $\in \ell^{2}(\nabla \mathcal{X})$ admitting a best $N$-term approximation converging with rate $s>0$ in the approximation class $\mathcal{A}_{\infty}^{s}\left(\ell^{2}\left(\nabla_{\mathcal{X}}\right)\right):=\left\{\mathbf{v} \in \ell^{2}\left(\nabla_{\mathcal{X}}\right):\|\mathbf{v}\|_{\mathcal{A}_{\infty}^{s}\left(\ell^{2}\left(\nabla_{\mathcal{X}}\right)\right)}<\infty\right\}$, where

$$
\|\mathbf{v}\|_{\mathcal{A}_{\infty}^{s}\left(\ell^{2}\left(\nabla_{\mathcal{X}}\right)\right)}:=\sup _{\varepsilon>0} \varepsilon\left[\min \left\{N \in \mathbb{N}_{0}:\left\|\mathbf{v}-\mathbf{v}_{N}\right\|_{\ell^{2}\left(\nabla_{\mathcal{X}}\right)} \leq \varepsilon\right\}\right]^{s},
$$

which consists of all $\mathbf{v} \in \ell^{2}\left(\nabla_{\mathcal{X}}\right)$ whose best $N$-term approximations converge to $\mathbf{v}$ with rate $s$; the symbol $\mathbb{N}_{0}:=\{0\} \cup \mathbb{N}$ signifies the set of all nonnegative integers.

Generally, best $N$-term approximations cannot be realized in practice, in particular not in situations where the vector $\mathbf{u}$ to be approximated is only defined implicitly as the solution of a bi-infinite matrix-vector problem, such as (5.1). We will now design, following [20], a space-time adaptive Galerkin discretization method for the infinitedimensional Fokker-Planck equation (4.5) that produces a sequence of approximations to $\mathbf{u}$, which, whenever $\mathbf{u} \in \mathcal{A}_{\infty}^{s}\left(\ell^{2}(\nabla \mathcal{X})\right)$ for some $s>0$, converge to $\mathbf{u}$ with this rate $s>0$ in computational complexity that is linear with respect to $N$, the cardinality of the support of the "active" coefficients in the computed, finitely supported approximation to $\mathbf{u}$.

\section{Adaptive Galerkin methods}

Let $s>0$ be such that $\mathbf{u} \in \mathcal{A}_{\infty}^{s}\left(\ell^{2}\left(\nabla_{\mathcal{X}}\right)\right)$. In [7] and [8], adaptive wavelet Galerkin methods for solving elliptic operator equations (5.1) were introduced; the methods considered in both papers are iterative methods. To be able to bound their complexity, one needs a suitable bound on the complexity of an approximate matrix-vector product in terms of the prescribed tolerance. We formalize this idea through the notion of $s^{*}$-admissibility.

Definition 7.1 The bi-infinite matrices $\mathbf{B} \in \mathcal{L}\left(\ell^{2}\left(\nabla_{\mathcal{X}}\right), \ell^{2}(\nabla \mathcal{y})\right), \mathbf{B}^{*} \in \mathcal{L}\left(\ell^{2}(\nabla \mathcal{Y})\right.$, $\left.\ell^{2}(\nabla \mathcal{X})\right)$ are $s^{*}$-admissible if there exist routines

$$
\operatorname{APPLY}_{\mathbf{B}}[\mathbf{w}, \varepsilon] \rightarrow \mathbf{z}, \quad \operatorname{APPLY}_{\mathbf{B}^{*}}[\tilde{\mathbf{w}}, \varepsilon] \rightarrow \tilde{\mathbf{z}}
$$

that yield, for any prescribed tolerance $\varepsilon>0$ and any finitely supported $\mathbf{w} \in \ell^{2}\left(\nabla_{\mathcal{X}}\right)$ and $\tilde{\boldsymbol{w}} \in \ell^{2}(\nabla \mathcal{Y})$, finitely supported vectors $\mathbf{z} \in \ell^{2}\left(\nabla_{\mathcal{Y}}\right)$ and $\tilde{\mathbf{z}} \in \ell^{2}\left(\nabla_{\mathcal{X}}\right)$ such that

$$
\|\mathbf{B w}-\mathbf{z}\|_{\ell^{2}\left(\nabla_{\mathcal{Y}}\right)}+\left\|\mathbf{B}^{*} \tilde{\mathbf{w}}-\tilde{\mathbf{z}}\right\|_{\ell^{2}\left(\nabla_{\mathcal{X}}\right)} \leq \varepsilon
$$


and for which, for any $\bar{s} \in\left(0, s^{*}\right)$, there exists an admissibility constant $a_{\boldsymbol{B}, \bar{s}}$ such that

$$
\# \operatorname{supp} \mathbf{z} \leq a_{\mathbf{B}, \bar{s}} \varepsilon^{-1 / \bar{s}}\|\mathbf{w}\|_{\mathcal{A}_{\infty}^{\bar{s}}\left(\ell^{2}\left(\nabla_{\mathcal{X}}\right)\right)}^{1 / \bar{s}},
$$

and likewise for $\tilde{\boldsymbol{z}}, \tilde{\boldsymbol{w}}$. The number of arithmetic operations and storage locations used by the call $\operatorname{APPLY}_{\boldsymbol{B}}[\boldsymbol{w}, \varepsilon]$ is bounded by some absolute multiple of

$$
a_{\mathbf{B}, \bar{s}} \varepsilon^{-1 / \bar{s}}\|\mathbf{w}\|_{\mathcal{A}_{\infty}^{\bar{s}}\left(\ell^{2}(\nabla \mathcal{X})\right)}^{1 / \bar{s}}+\# \operatorname{supp} \mathbf{w}+1
$$

The design of $\operatorname{APPLY}_{\mathbf{B}}[\mathbf{w}, \varepsilon]$ and $\operatorname{APPLY}_{\mathbf{B}^{*}}[\tilde{\mathbf{w}}, \varepsilon]$ for the system matrices $\mathbf{B}$ and $\mathbf{B}^{*}$ arising from the infinite-dimensional Fokker-Planck equation (4.5) is the major technical building block in the adaptive Galerkin discretization of (4.5).

In order to approximate $\mathbf{u}$ one should be able to approximate $\mathbf{f}^{*}$. Throughout this paper we shall assume the availability of the following routine.

$\mathbf{R H S}_{\mathbf{f} *}[\varepsilon] \rightarrow \mathbf{f}_{\varepsilon}^{*}:$ For given $\varepsilon>0$, the routine yields a finitely supported $\mathbf{f}_{\varepsilon}^{*}$ $\in \ell^{2}(\nabla \mathcal{X})$ with

$$
\left\|\mathbf{f}^{*}-\mathbf{f}_{\varepsilon}^{*}\right\|_{\ell^{2}\left(\nabla_{\mathcal{X}}\right)} \leq \varepsilon \text { and } \# \operatorname{supp} \mathbf{f}_{\varepsilon}^{*} \lesssim \min \left\{N:\left\|\mathbf{f}^{*}-\mathbf{f}_{N}^{*}\right\| \leq \varepsilon\right\}
$$

with the number of arithmetic operations and storage locations used by the call $\mathbf{R H S}_{\mathbf{f}}^{*}[\varepsilon]$ being bounded by some absolute multiple of \#supp $\mathbf{f}_{\varepsilon}^{*}+1$.

The availability of the routines $\mathbf{A P P L Y}_{\mathbf{B}}$ and $\mathbf{R H S}_{\mathbf{f}}$ has the following implications.

Proposition 7.2 Let $\mathbf{B}$ in (5.1) be $s^{*}$-admissible. Then, for any $\bar{s} \in\left(0, s^{*}\right)$, we have that

$$
\|\mathbf{B}\|_{\mathcal{A}_{\infty}^{\bar{s}}\left(\ell^{2}(\nabla \mathcal{X})\right) \rightarrow \mathcal{A}_{\infty}^{\bar{s}}\left(\ell^{2}(\nabla \mathcal{Y})\right)} \leq a_{\mathbf{B}, \bar{s}}^{\bar{s}}
$$

For $\mathbf{z}_{\varepsilon}:=\operatorname{APPLY}_{\mathbf{B}}[\mathbf{w}, \varepsilon]$, we have that

$$
\left\|\mathbf{z}_{\varepsilon}\right\|_{\mathcal{A}_{\infty}^{\bar{s}}\left(\ell^{2}\left(\nabla_{\mathcal{Y}}\right)\right)} \leq a_{\mathbf{B}, \bar{s}}^{\bar{s}}\|\mathbf{w}\|_{\mathcal{A}_{\infty}^{\bar{s}}\left(\ell^{2}\left(\nabla_{\mathcal{X}}\right)\right)}
$$

Analogous statements hold for $\mathbf{B}^{*}$ in (5.1).

A proof of Proposition 7.2 can be given along the lines of the arguments presented in [7,8]. Using the definition of $\mathcal{A}_{\infty}^{s}\left(\ell^{2}\left(\nabla_{\mathcal{Y}}\right)\right)$ and the properties of $\mathbf{R H S}_{\mathbf{f}}$, we have the following corollary.

Corollary 7.3 Suppose that, in (5.1), $\mathbf{B}^{*}$ is $s^{*}$-admissible and $\mathbf{u} \in \mathcal{A}_{\infty}^{s}\left(\ell^{2}(\nabla \mathcal{y})\right)$ for $s<s^{*}$; then, for $\mathbf{f}_{\varepsilon}^{*}=\mathbf{R H S}_{\mathbf{f}^{*}}[\varepsilon]$,

$$
\# \operatorname{supp} \mathbf{f}_{\varepsilon}^{*} \lesssim a_{\mathbf{B}^{*}, s} \varepsilon^{-1 / s}\|\mathbf{u}\|_{\mathcal{A}_{\infty}^{s}\left(\ell^{2}(\nabla \mathcal{Y})\right)}^{1 / s}
$$


with the number of arithmetic operations and storage locations used by the call $\mathbf{R H S}_{\mathbf{f} *}[\varepsilon]$ being bounded by some absolute multiple of

$$
a_{\mathbf{B}^{*}, s} \varepsilon^{-1 / s}\|\mathbf{u}\|_{\mathcal{A}_{\infty}^{s}\left(\ell^{2}(\nabla \mathcal{Y})\right)}^{1 / s}+1 .
$$

Remark 7.4 Besides $\left\|\mathbf{f}^{*}-\mathbf{f}_{\varepsilon}^{*}\right\|_{\ell^{2}\left(\nabla_{\mathcal{Y}}\right)} \leq \varepsilon$, the complexity bounds in Corollary 7.3, with $a_{\mathbf{B}^{*}, s}$ signifying a constant that depends only on $\mathbf{B}^{*}$ and $s$, are essential for the use of $\mathbf{R H S}_{\mathbf{f}} *$ in the adaptive Galerkin methods.

The following corollary of Proposition 7.2 from [20] can be used for example for the construction of valid APPLY and RHS routines in case the adaptive Galerkin algorithms are applied to a preconditioned system.

Corollary 7.5 Suppose that $\mathbf{B}^{*} \in \mathcal{L}\left(\ell^{2}\left(\nabla_{\mathcal{Y}}\right), \ell^{2}\left(\nabla_{\mathcal{X}}\right)\right), \mathbf{C} \in \mathcal{L}\left(\ell^{2}\left(\nabla_{\mathcal{X}}\right), \ell^{2}\left(\nabla_{\mathcal{Z}}\right)\right)$ are both $s^{*}$-admissible; then, so is $\mathbf{C B}^{*} \in \mathcal{L}\left(\ell^{2}\left(\nabla_{\mathcal{Y}}\right), \ell^{2}\left(\nabla_{\mathcal{Z}}\right)\right)$. A valid routine $\operatorname{APPLY}_{\mathbf{C B}^{*}}$ is

$$
[\mathbf{w}, \varepsilon] \mapsto \operatorname{APPLY}_{\mathbf{C}}\left[\operatorname{APPLY}_{\mathbf{B}^{*}}[\mathbf{w}, \varepsilon /(2\|\mathbf{C}\|)], \varepsilon / 2\right]
$$

with admissibility constant $a_{\mathbf{C B}^{*}, \bar{s}} \lesssim a_{\mathbf{B}^{*}, \bar{s}}\left(\|\mathbf{C}\|^{1 / \bar{s}}+a_{\mathbf{C}, \bar{s}}\right)$ for $\bar{s} \in\left(0, s^{*}\right)$.

For some $s^{*}>s$, let $\mathbf{C} \in \mathcal{L}\left(\ell^{2}\left(\nabla_{\mathcal{Y}}\right), \ell^{2}\left(\nabla_{\mathcal{Z}}\right)\right)$ be $s^{*}$-admissible. Then, for

$$
\mathbf{R H S}_{\mathbf{C f} *}[\varepsilon]:=\operatorname{APPLY}_{\mathbf{C}}\left[\mathbf{R H S}_{\mathbf{f} *}[\varepsilon /(2\|\mathbf{C}\|)], \varepsilon / 2\right],
$$

we have that

$$
\# \operatorname{supp} \mathbf{R H S}_{\mathbf{C f} *}[\varepsilon] \lesssim a_{\mathbf{B}^{*}, s}\left(\|\mathbf{C}\|^{1 / s}+a_{\mathbf{C}, s}\right) \varepsilon^{-1 / s}\|\mathbf{u}\|_{\mathcal{A}_{\infty}^{s}\left(\ell^{2}(\nabla \mathcal{Y})\right)}^{1 / s}
$$

and $\left\|\mathbf{C f}^{*}-\mathbf{R H S}_{\mathbf{C f} *}[\varepsilon]\right\|_{\ell^{2}\left(\nabla_{\mathcal{Z}}\right)} \leq \varepsilon$, with the number of arithmetic operations and storage locations used by the call $\mathbf{R} \mathbf{H} \mathbf{S}_{\mathbf{C f}}[\varepsilon]$ being bounded by some absolute multiple of

$$
a_{\mathbf{B}^{*}, s}\left(\|\mathbf{C}\|^{1 / s}+a_{\mathbf{C}, s}\right) \varepsilon^{-1 / s}\|\mathbf{u}\|_{\mathcal{A}_{\infty}^{s}\left(\ell^{2}\left(\nabla_{\mathcal{X}}\right)\right)}^{1 / s}+1
$$

Remark 7.6 The properties of $\mathbf{R H S}_{\mathbf{C f} *}$ stated in Corollary 7.5 show that $\mathbf{R H S} \mathbf{C f}_{\mathbf{C}}$ is a valid routine for approximating $\mathbf{C} \mathbf{f}^{*}$ in the sense of Remark 7.4.

In the particular case when $\mathbf{B}$ is symmetric positive definite, i.e., $\nabla \mathcal{X}=\nabla \mathcal{y}$ and $\mathbf{B}=\mathbf{B}^{*}>0$, the two Galerkin methods considered in the papers [7,8] were shown to be quasioptimal in the following sense.

Theorem 7.7 Suppose that, in (5.1), $\mathbf{B}^{*}$ is $s^{*}$-admissible; then, for any $\varepsilon>0$ the two adaptive Galerkin methods from [7,8] produce an approximation $\mathbf{u}_{\varepsilon}$ to $\mathbf{u}$ with $\left\|\mathbf{u}-\mathbf{u}_{\varepsilon}\right\|_{\ell^{2}(\nabla y)} \leq \varepsilon$. Suppose that, in (5.1), for some $s>0$ we have that $\mathbf{u} \in$ $\mathcal{A}_{\infty}^{s}\left(\ell^{2}(\nabla \mathcal{Y})\right)$; then \# $\operatorname{supp} \mathbf{u}_{\varepsilon} \lesssim \varepsilon^{-1 / s}\|\mathbf{u}\|_{\mathcal{A}_{\infty}^{s}\left(\ell^{2}(\nabla \mathcal{Y})\right)}^{1 / s}$ and if, moreover, $s<s^{*}$, then the 
number of arithmetic operations and storage locations required by a call of either of these adaptive solvers with tolerance $\varepsilon>0$ is bounded by some multiple of

$$
\varepsilon^{-1 / s}\left(1+a_{\mathbf{B}, s}\right)\|\mathbf{u}\|_{\mathcal{A}_{\infty}^{s}\left(\ell^{2}\left(\nabla_{\mathcal{Y}}\right)\right)}^{1 / s}+1
$$

The multiples depend on s only when s tends to 0 or $\infty$, and on $\|\mathbf{B}\|$ and $\left\|\mathbf{B}^{-1}\right\|$ when they tend to infinity.

The method from [8] is based on a damped Richardson iteration for the approximate solution of $\mathbf{B u}=\mathbf{f}$, where the required residual computations are approximated using calls of $\mathbf{A P P L Y}_{\mathbf{B}}$ and $\mathbf{R H S}_{\mathbf{f}}$ within tolerances that decrease linearly with the iteration counter.

The method from [7] produces a sequence $\Xi_{0} \subset \Xi_{1} \subset \cdots \subset \nabla_{\mathcal{X}}$, together with a corresponding sequence of (approximate) finitely supported Galerkin solutions $\mathbf{u}_{i} \in \ell^{2}\left(\Xi_{i}\right)$. The coefficients of approximate residuals $\mathbf{f}-\mathbf{B} \mathbf{u}_{i}$ are used to steer the expansion of the sets $\left\{\Xi_{i}\right\}_{i \geq 0}$.

Both methods rely on a recurrent coarsening of the approximation vectors, where small coefficients are removed in order to keep an optimal balance between accuracy and support length; in [13], a modification of the algorithm introduced in [7] was proposed, which does not require the coarsening step.

The $s^{*}$-admissibility of $\mathbf{B}$ can be expected, since the structure of $\mathbf{B}$ in the multiresolution Riesz-bases in $[0, T]$ and in Wiener-Hermite polynomial chaos bases of $L^{2}(H ; \mu)$ is, as we will show in what follows, close to that of a computable sparse matrix.

Definition 7.8 $\mathbf{B} \in \mathcal{L}\left(\ell^{2}\left(\nabla_{\mathcal{X}}\right), \ell^{2}(\nabla \mathcal{Y})\right)$ is $s^{*}$-computable if, for each $N \in \mathbb{N}$, there exists a $\mathbf{B}^{[N]} \in \mathcal{L}\left(\ell^{2}\left(\nabla_{\mathcal{X}}\right), \ell^{2}(\nabla \mathcal{Y})\right)$ having in each column at most $N$ nonzero entries whose joint computation takes an absolute multiple of $N$ operations, such that the computability constants

$$
c_{\mathbf{B}, \bar{s}}:=\sup _{N \in \mathbb{N}} N\left\|\mathbf{B}-\mathbf{B}^{[N]}\right\|_{\ell^{2}(\nabla \mathcal{X}) \rightarrow \ell^{2}(\nabla \mathcal{Y})}^{1 / \bar{s}}
$$

are finite for any $\bar{s} \in\left(0, s^{*}\right)$. The notion of $s^{*}$-computability of $\mathbf{B}^{*}$ is defined analogously.

Proposition 7.9 An $s^{*}$-computable $\mathbf{B}$ is $s^{*}$-admissible. Moreover, for $\bar{s}<s^{*}, a_{\mathbf{B}, \bar{s}} \lesssim$ $c_{\mathbf{B}, \bar{s}}$, where the constant in this bound depends only on $\bar{s} \downarrow 0, \bar{s} \uparrow s^{*}$, and on $\|\mathbf{B}\| \rightarrow \infty$.

This is proved by constructing a suitable $\mathbf{A P P L Y}$ B routine as in [7, §6.4] (a log factor in the complexity bound there due to sorting was removed later by application of an approximate sorting, see [12] and the references therein).

Remark 7.10 Theorem 7.7 requires that $\mathbf{B}$ is $s^{*}$-admissible for an $s^{*}>s$ when $\mathbf{u} \in$ $\mathcal{A}_{\infty}^{s}\left(\ell^{2}(\nabla \mathcal{X})\right)$. Generally this value of $s$ is unknown, and so the condition on $s^{*}$ should be interpreted in the sense that $s^{*}$ has to be larger than any $s$ for which membership of the solution $\mathbf{u}$ in $\mathcal{A}_{\infty}^{s}\left(\ell^{2}\left(\nabla_{\mathcal{X}}\right)\right)$ can be expected. 
The approach from [8] applies also to the saddle-point variational principle (4.5) whenever one has a linearly convergent stationary iterative scheme available for the matrix-vector problem $\mathbf{B}^{*} \mathbf{u}=\mathbf{f}^{*}$. There is, unfortunately, no such scheme available for a general boundedly invertible $\mathbf{B}^{*}$. In particular, for the stiffness matrices $\mathbf{B}^{*}$ resulting from the space-time saddle-point formulation (4.5) of the infinite-dimensional FokkerPlanck equation no directly applicable scheme is available. In [8], adaptive Galerkin discretizations for such problems are proposed for the normal equation

$$
\mathbf{B B}^{*} \mathbf{u}=\mathbf{B f}^{*} \text {. }
$$

By Theorem 4.3, the operator $\mathbf{B B}^{*} \in \mathcal{L}\left(\ell^{2}(\nabla \mathcal{y}), \ell^{2}(\nabla \mathcal{Y})\right)$ is boundedly invertible, symmetric positive definite, with

$$
\begin{aligned}
&\left\|\mathbf{B B}^{*}\right\|_{\ell^{2}(\nabla \mathcal{Y}) \rightarrow \ell^{2}(\nabla \mathcal{Y})} \leq\left\|\mathbf{B}^{*}\right\|_{\ell^{2}(\nabla \mathcal{Y}) \rightarrow \ell^{2}\left(\nabla_{\mathcal{X}}\right)}^{2},\left\|\left(\mathbf{B B}^{*}\right)^{-1}\right\|_{\ell^{2}(\nabla \mathcal{Y}) \rightarrow \ell^{2}(\nabla \mathcal{Y})} \\
& \leq\left\|\left(\mathbf{B}^{*}\right)^{-1}\right\|_{\ell^{2}(\nabla \mathcal{X}) \rightarrow \ell^{2}(\nabla \mathcal{Y})}^{2} .
\end{aligned}
$$

Now let $\mathbf{u} \in \mathcal{A}_{\infty}^{s}\left(\ell^{2}\left(\nabla_{\mathcal{X}}\right)\right)$, and for some $s^{*}>s$, let $\mathbf{B}$ and $\mathbf{B}^{*}$ be $s^{*}$-admissible. By Corollary 7.5, with $\mathbf{B}^{*}$ in place of $\mathbf{C}$, a valid $\mathbf{R H S} \mathbf{S}_{\mathbf{B}} *$ routine is given by (7.2), and $\mathbf{B B}^{*}$ is $s^{*}$-admissible with a valid $\mathbf{A P P L Y} \mathbf{B B}^{*}$ routine given by (7.1). In the context of (4.5), one execution of $\mathbf{A P P L Y}_{\mathbf{B B}}$ * corresponds to one (approximate) sweep over the "primal" problem, followed by one (approximate) sweep over the dual (adjoint) problem, respectively. A combination of Theorem 7.7 and Corollary 7.5 yields the following result, obtained in [20], which will be the basis for our Wiener-Hermite polynomial chaos discretization.

Theorem 7.11 For any $\varepsilon>0$, the adaptive wavelet methods from [8] or from [7] and [13] applied to the normal equations (7.4) using the above $\mathbf{A P P L Y} \mathbf{B B}_{\mathbf{B}}$ and $\mathbf{R H} \mathbf{S}_{\mathbf{B} f^{*}}$ routines produce an approximation $\mathbf{u}_{\varepsilon}$ to $\mathbf{u}$ with $\left\|\mathbf{u}-\mathbf{u}_{\varepsilon}\right\|_{\ell^{2}(\nabla \mathcal{Y})} \leq \varepsilon$.

Suppose that for some $s>0, \mathbf{u} \in \mathcal{A}_{\infty}^{s}\left(\ell^{2}(\nabla \mathcal{Y})\right)$; then \#supp $\mathbf{u}_{\varepsilon} \lesssim \varepsilon^{-1 / s}$ $\|\mathbf{u}\|_{\mathcal{A}_{\infty}^{s}\left(\ell^{2}\left(\nabla_{\mathcal{Y}}\right)\right)}^{1 / s}$, with the constant in this bound only being dependent on $s$ when it tends to 0 or $\infty$, and on $\left\|\mathbf{B}^{*}\right\|$ and $\left\|\left(\mathbf{B}^{*}\right)^{-1}\right\|$ when they tend to infinity.

Suppose that $s<s^{*}$; then, the number of arithmetic operations and storage locations required by a call of either of these adaptive wavelet methods with tolerance $\varepsilon$ is bounded by some multiple of

$$
1+\varepsilon^{-1 / s}\left(1+a_{\mathbf{B}^{*}, s}\left(1+a_{\mathbf{B}, s}\right)\right)\|\mathbf{u}\|_{\mathcal{A}_{\infty}^{s}\left(\ell^{2}(\nabla \mathcal{Y})\right)}^{1 / s},
$$

where this multiple only depends on $s$ when it tends to 0 or $\infty$, and on $\left\|\mathbf{B}^{*}\right\|$ and $\left\|\left(\mathbf{B}^{*}\right)^{-1}\right\|$ when they tend to infinity. 


\section{Infinite-dimensional Fokker-Planck equation as bi-infinite matrix vector equation}

We apply the foregoing abstract concepts to the space-time variational formulation (4.5) of the infinite-dimensional Fokker-Planck equation. To construct Riesz bases for $\mathcal{X}_{0,\{T\}}$ and $\mathcal{Y}$, we use that

$$
\mathcal{X}_{0,\{T\}}=\left(\mathrm{L}^{2}(I) \otimes \mathcal{V}\right) \cap\left(\mathrm{H}_{0,\{T\}}^{1}(I) \otimes \mathcal{V}^{*}\right) \quad \text { and } \quad \mathcal{Y}=\mathrm{L}^{2}(I) \otimes \mathcal{V}
$$

with the spaces $\mathcal{X}_{0,\{T\}}$ as defined in (4.4), and $\mathcal{Y}=\mathrm{L}^{2}(0, T ; \mathcal{V})$; recall that $\mathcal{H}$ $=\mathrm{L}^{2}(D, \mu)$ and $\mathcal{V}=\mathrm{W}^{1,2}(D, \mu)$, with $\mathcal{V}$ continuously, densely and compactly embedded into $\mathcal{H}$. Let

$$
\Upsilon=\left\{H_{\gamma}: \gamma \in \Gamma\right\} \subset \mathcal{V}, \quad \text { with } H_{\gamma}(q)=\prod_{k \geq 1} H_{\gamma_{k}}\left(\frac{q_{k}}{\sqrt{\lambda_{k}}}\right),
$$

denote the Wiener-Hermite polynomial chaos basis (3.3). By Theorem 3.2, $\Upsilon$ is a normalized Riesz basis for $\left[\mathrm{L}^{2}(H, \mu)\right]^{d}$, which, when rescaled by the scaling sequence $\left\{\left(\left\langle\gamma, \lambda^{-1}\right\rangle_{*}\right)^{-1 / 2}: \gamma \in \Gamma\right\}$ in $\mathcal{V}$ and by the scaling sequence $\left\{\left(\left\langle\gamma, \lambda^{-1}\right\rangle_{*}\right)^{1 / 2}: \gamma \in \Gamma\right\}$ in $\mathcal{V}^{*}$, is a Riesz basis for $\mathcal{V}$ and $\mathcal{V}^{*}$, respectively; here the scaling factors are defined by

$$
\left\langle\gamma, \lambda^{-1}\right\rangle_{*}:=\left\{\begin{array}{cl}
\left\langle\gamma, \lambda^{-1}\right\rangle & \text { if } \gamma \in \Gamma \backslash\{0\} \\
1 & \text { if } \gamma=0
\end{array}\right.
$$

see (3.6), (3.7) and also (9.1), (9.2) below. For the time interval $I=(0, T)$, we denote by

$$
\Theta=\left\{\theta_{\lambda}: \lambda \in \nabla_{t}\right\} \subset \mathrm{H}_{0,\{T\}}^{1}(I)
$$

a collection of functions indexed by the countable set $\nabla_{t}$ that is a normalized Riesz basis for $\mathrm{L}^{2}(I)$, which, when rescaled in $\mathrm{H}^{1}(I)$, is a Riesz basis for $\mathrm{H}_{0,\{T\}}^{1}(I)$. It then follows from [14, Prop. 1 and 2] that the collection $\Theta \otimes \Upsilon$, normalized in $\mathcal{X}$, i.e., the collection

$$
\left\{(t, q) \mapsto \frac{\theta_{\lambda}(t) H_{\gamma}(q)}{\sqrt{\left\langle\gamma, \lambda^{-1}\right\rangle_{*}+\left\|\theta_{\lambda}\right\|_{\mathrm{H}^{1}(I)}^{2} /\left\langle\gamma, \lambda^{-1}\right\rangle_{*}}}:(\lambda, \gamma) \in \nabla_{\mathcal{X}}:=\nabla_{t} \times \Gamma\right\},
$$

is a Riesz basis for $\mathcal{X}_{0,\{T\}}$, and that $\Theta \otimes \Upsilon$ normalized in $\mathcal{Y}$, i.e., the collection

$$
\left\{(t, q) \mapsto \frac{\theta_{\lambda}(t) H_{\gamma}(q)}{\left\|H_{\gamma}\right\|_{\mathcal{V}}}:(\lambda, \gamma) \in \nabla_{\mathcal{Y}}:=\nabla_{t} \times \Gamma\right\}
$$

is a Riesz basis for $\mathcal{Y}$. Moreover, denoting the Riesz basis for $\mathcal{V}^{*}$ consisting of the collection $\Upsilon$ normalized in $\mathcal{V}^{*}$ by $[\Upsilon] \mathcal{V}^{*}$, and similarly for the other collections and 
spaces, with the notations introduced in section 5 , we have that

$$
\begin{aligned}
& \Lambda_{[\Theta \otimes \Upsilon]_{\mathcal{X}}}^{\mathcal{X}} \leq \max \left(\Lambda_{\Theta}^{\mathrm{L}^{2}(I)} \Lambda_{[\Upsilon] \mathcal{V}}^{\mathcal{V}}, \Lambda_{[\Theta]_{\mathrm{H}^{1}(I)}}^{\mathrm{H}^{1}(I)} \Lambda_{[\Upsilon]_{\mathcal{V}^{*}}}^{\mathcal{V}^{*}}\right) \\
& \lambda_{[\Theta \otimes \Upsilon]_{\mathcal{X}}}^{\mathcal{X}} \geq \min \left(\lambda_{\Theta}^{\mathrm{L}^{2}(I)} \lambda_{[\Upsilon]_{\mathcal{V}}}^{\mathcal{V}}, \lambda_{[\Theta]_{\mathrm{H}^{1}(I)}}^{\mathrm{H}^{1}(I)} \lambda_{[\Upsilon]_{\mathcal{V}^{*}}}^{\mathcal{V}^{*}}\right), \\
& \Lambda_{[\Theta \otimes \Upsilon] \mathcal{Y}}^{\mathcal{Y}} \leq \Lambda_{\Theta}^{L^{2}(I)} \Lambda_{[\Upsilon] \mathcal{V}}^{\mathcal{V}}, \\
& \lambda_{[\Theta \otimes \Upsilon] \mathcal{Y}}^{\mathcal{Y}} \geq \lambda_{\Theta}^{L^{2}(I)} \lambda_{[\Upsilon] \mathcal{V}}^{\mathcal{V}} .
\end{aligned}
$$

Denoting by $\|\Upsilon\|_{\mathcal{V}}$ the infinite diagonal matrix with diagonal entries $\left\|H_{\gamma}\right\| \mathcal{V}$ where $\gamma \in \Gamma$, and similarly for the other collections and spaces, the stiffness or system matrix $\mathbf{B}^{*}$ corresponding to the variational form (4.5) and the Riesz bases $[\Theta \otimes \Upsilon] \mathcal{Y},[\Theta \otimes \Upsilon] \mathcal{X}$ for $\mathcal{Y}$ and $\mathcal{X}_{0,\{T\}}$ is given by the bi-infinite matrix

$$
\begin{aligned}
\mathbf{B}^{*}= & \mathfrak{B}^{*}\left([\Theta \otimes \Upsilon] \mathcal{Y},[\Theta \otimes \Upsilon]_{\mathcal{X}}\right) \\
= & {\left[\mathrm{Id}_{t} \otimes\|\Upsilon\|_{\mathcal{V}}^{-1}\right] \circ\left[-\left(\Theta, \Theta^{\prime}\right)_{\mathrm{L}^{2}(I)} \otimes(\Upsilon, \Upsilon)_{\mathcal{H}}+\int_{I} \mathfrak{a}(\Theta \otimes \Upsilon, \Theta \otimes \Upsilon) \mathrm{d} t\right] \circ } \\
& {\left[\|\Theta \otimes \Upsilon\|_{\mathcal{X}}^{-1}\right], }
\end{aligned}
$$

where $\operatorname{Id}_{t}$ denotes the identity operator with respect to the $t$ variable and the symbol o signifies composition of operators.

Writing the solution $u$ of (4.5) as $u=\mathbf{u}^{\top}[\Theta \otimes \Sigma] \mathcal{X}$, we deduce that $\mathbf{u}$ is the solution of the bi-infinite matrix-vector equation $\mathbf{B}^{*} \mathbf{u}=\mathbf{f}^{*}$ with

$$
\mathbf{f}^{*}:=\left[\left(\widehat{\psi}_{0},[\Upsilon]_{\mathcal{H}}\right)_{\mathcal{H}}\right] .
$$

By introducing the infinite diagonal matrices

$$
\mathbf{D}_{1}:=\left(\|\Theta\|_{\mathrm{H}^{1}(I)} \otimes\|\Upsilon\|_{\mathcal{V}^{*}}\right)\|\Theta \otimes \Upsilon\|_{\mathcal{X}}^{-1} \quad \text { and } \quad \mathbf{D}_{2}:=\left(\operatorname{Id}_{t} \otimes\|\Upsilon\|_{\mathcal{V}}\right)\|\Theta \otimes \Upsilon\|_{\mathcal{X}}^{-1} \text {, }
$$

both having diagonal entries in modulus less than 1, the bi-infinite matrix operator $\mathbf{B}^{*}$ can be written as

$$
\begin{aligned}
\mathbf{B}^{*}= & {\left[\left(-[\Theta]_{\mathrm{H}_{0,\{T\}}^{1}(I)^{*}}, \Theta^{\prime}\right)_{\mathrm{L}^{2}(I)} \otimes\left([\Upsilon] \mathcal{V}^{*},[\Upsilon] \mathcal{V}\right)_{\mathcal{H}} \mathbf{D}_{1}\right.} \\
& \left.+\int_{I} \mathfrak{a}(\Theta \otimes[\Upsilon] \mathcal{V}, \Theta \otimes[\Upsilon] \mathcal{V}) \mathrm{d} t \mathbf{D}_{2}\right]
\end{aligned}
$$

\section{$9 s^{*}$-admissibility of $\mathbf{B}$ and $\mathbf{B}^{*}$ from (8.7) and of its adjoint}

By Theorem 7.9, the $s^{*}$-admissibility of $\mathbf{B}$ and $\mathbf{B}^{*}$ follows from their $s^{*}$-computability. To verify the $s^{*}$-computability of tensor products of possibly bi-infinite matrices, it suffices to analyze the $s^{*}$-computability of the factors, according to the following result from $[20,16]$. 
Proposition 9.1 Let, for some $s^{*}>0$, $\mathbf{D}$ and $\mathbf{E}$ be $s^{*}$-computable. Then,

(a) $\mathbf{D} \otimes \mathbf{E}$ is $s^{*}$-computable with computability constant satisfying, for $0<\bar{s}<\tilde{s}<$ $s^{*}, c_{\mathbf{D} \otimes \mathbf{E}, \bar{s}} \lesssim\left(c_{\mathbf{D}, \tilde{s}} c_{\mathbf{E}, \tilde{s}}\right)^{\tilde{s} / \bar{s}} ;$ and

(b) for any $\varepsilon \in\left(0, s^{*}\right), \mathbf{D} \otimes \mathbf{E} i s\left(s^{*}-\varepsilon\right)$-computable, with computability constant $c_{\mathbf{D} \otimes \mathbf{E}, \bar{s}}$ satisfying, for $0<\bar{s}<s^{*}-\varepsilon<\tilde{s}<s^{*}, c_{\mathbf{D} \otimes \mathbf{E}, \bar{s}} \lesssim$ $\max \left(c_{\mathbf{D}, \tilde{s}}, 1\right) \max \left(c_{\mathbf{E}, \tilde{s}}, 1\right)$.

The constants absorbed in the $\lesssim$ symbol in the bounds on the computability constants in (a) and (b) are only dependent on $\tilde{s} \downarrow 0, \tilde{s} \rightarrow \infty$ and $\tilde{s}-\bar{s} \downarrow 0$.

In view of the representation (8.7) of $\mathbf{B}^{*}$, using Corollary 7.5 and Proposition 9.1, part (a), for proving $s^{*}$-admissibility of $\mathbf{B}$ and $\mathbf{B}^{*}$ it suffices to show that $(\Upsilon, \Upsilon)_{\mathcal{H}}$ and $\int_{I} \mathfrak{a}(\Theta \otimes[\Upsilon] \mathcal{V}, \Theta \otimes[\Upsilon] \mathcal{V}) \mathrm{d} t$ and its adjoint are $s^{*}$-admissible. To prove this, by Theorem 7.9 we need to verify that these objects are $s^{*}$-computable. This will follow from the fact that $\left([\Theta]_{\mathrm{H}^{1}(I)}^{\prime}, \Theta\right)_{\mathrm{L}^{2}(I)},\left(\Theta,[\Theta]_{\mathrm{H}^{1}(I)}^{\prime}\right)_{\mathrm{L}^{2}(I)},\left([\Upsilon]_{\mathcal{V}^{*}},[\Upsilon] \mathcal{V}\right)_{\mathcal{H}}$ and $\left([\Upsilon] \mathcal{V},[\Upsilon] \mathcal{V}^{*}\right) \mathcal{H}$ are $s^{*}$-computable and, in view of the definition of $\mathfrak{a}(\cdot, \cdot)$ in (4.6), from a sparsity assumption on the coefficient matrix $\mathbf{A}$.

\subsection{Choice of Riesz bases $\Theta$ and $\Upsilon$}

We have already assumed that $\Theta=\left\{\theta_{\lambda}: \lambda \in \nabla_{t}\right\}$ is a normalized Riesz basis of $\mathrm{L}^{2}(I)$ that, when rescaled in $\mathrm{H}^{1}(I)$ is a Riesz basis for $\mathrm{H}_{0,\{T\}}^{1}(I)$. We will now select the basis $\Theta$ to be a wavelet basis that satisfies certain additional assumptions. Specifically, we shall assume that the basis functions $\theta_{\lambda}$ of $\Theta$ are:

(t1) local, i.e., $\sup _{x \in[0,1], \ell \in \mathbb{N}_{0}} \#\left\{|\lambda|=\ell: x \in \operatorname{supp} \theta_{\lambda}\right\}<\infty$ and $\operatorname{supp} \theta_{\lambda} \lesssim 2^{-|\lambda|}$;

(t2) piecewise polynomial of degree $d_{t}$, where by "piecewise" we mean that the singular support consists of a set of points whose cardinality is uniformly bounded, $|\lambda|$

(t3) globally continuous, specifically $\left\|\theta_{\lambda}\right\|_{W_{\infty}^{k}(0,1)} \lesssim 2^{|\lambda|\left(\frac{1}{2}+k\right)}$ for $k \in\{0,1\}$;

(t4) for $|\lambda|>0$, have $\tilde{d}_{t} \geq d_{t}$ vanishing moments.

The assumptions (t1)-(t4) can be met by spline wavelet constructions (see, e.g. [6] and the references therein).

As a Riesz basis in $D$ we choose the (countable) "polynomial chaos" basis $\Upsilon$ $=\left\{H_{\gamma}: \gamma \in \Gamma\right\}$. According to Theorem 3.2, $\Upsilon$ is an orthonormal Riesz basis of $\mathrm{L}^{2}(D, \mu)$ that, rescaled in $\mathcal{V}=\mathrm{W}^{1,2}(D, \mu)$ or its dual $\mathcal{V}^{*}$, is a Riesz basis for these spaces, respectively. Indeed, on denoting by $\mathbf{D}^{\lambda}$ the diagonal matrix

$$
\mathbf{D}^{\lambda}=\operatorname{diag}\left\{\left(\left\langle\gamma, \lambda^{-1}\right\rangle_{*}\right)^{1 / 2}: \gamma \in \Gamma\right\}
$$

we observe that the diagonal entries of $\mathbf{D}^{\lambda}$ relate to the $\mathcal{V}$ and $\mathcal{V}^{*}$ norms of $[\Upsilon]_{\mathcal{H}}$ as follows:

$$
\mathbf{D}^{\lambda} \simeq\left\|[\Upsilon]_{\mathcal{H}}\right\| \mathcal{V}, \quad\left(\mathbf{D}^{\lambda}\right)^{-1} \simeq\left\|[\Upsilon]_{\mathcal{H}}\right\|_{\mathcal{V}^{*}}
$$


Therefore, the collection $[\Upsilon] \mathcal{V}:=\left(\mathbf{D}^{\lambda}\right)^{-1}[\Upsilon]_{\mathcal{H}}=\left\{\left(D_{\gamma}^{\lambda}\right)^{-1} H_{\gamma}: \gamma \in \Gamma\right\}$ is a Riesz basis of $\mathcal{V}=\mathrm{W}^{1,2}(D, \mu)$. This follows readily from the $\mathrm{L}^{2}(D, \mu)$ orthonormality of $\Upsilon$ and from the identity (cf. [10, (9.2.11)])

$$
\mathrm{D}_{k} H_{\gamma}=\gamma_{k}^{1 / 2} \lambda_{k}^{-1 / 2} H_{\gamma_{k}-1}\left(W_{e_{k}}\right) H_{\gamma}^{(k)}, \quad \text { for } k=1,2, \ldots \quad \text { and } \quad \gamma \in \Gamma \text {, }
$$

with the notational conventions $H_{-1}\left(W_{e_{k}}\right):=0$ and $H_{\gamma}^{(k)}:=\prod_{j \neq k} H_{\gamma_{j}}$ for $\gamma \in \Gamma$. By duality, the collection $[\Upsilon]_{\mathcal{V}^{*}}:=\mathbf{D}^{\lambda}[\Upsilon]_{\mathcal{H}}$ is a Riesz basis of $\mathcal{V}^{*}$.

$9.2 s^{*}$-computability of $\left([\Theta]_{\mathrm{H}^{1}(I)}^{\prime}, \Theta\right)_{\mathrm{L}^{2}(I)}$ and of its adjoint

By (t1), (t2) and (t4), for each $\lambda \in \nabla_{t}$ and $\ell \in \mathbb{N}_{0}$, the number of $\mu \in \nabla_{t}$ with $|\mu|=\ell$ and $\int_{I} \theta_{\lambda}^{\prime} \theta_{\mu} \mathrm{d} t \neq 0$ or $\int_{I} \theta_{\mu}^{\prime} \theta_{\lambda} \mathrm{d} t \neq 0$ is bounded, uniformly in $\lambda$ and $\ell$. Indeed, $\int_{I} \theta_{\lambda}^{\prime} \theta_{\mu} \mathrm{d} t$ can only be nonzero when $\theta_{\mu}$ does not vanish on the singular support of $\theta_{\lambda}$, and using integration by parts, $\int_{I} \theta_{\mu}^{\prime} \theta_{\lambda} \mathrm{d} t$ can only be nonzero when $\theta_{\mu}$ does not vanish on the singular support of $\theta_{\lambda}$ or at $\partial I$.

As a consequence of $\Theta$ being of degree $d_{t} \geq 1$ we have that

$$
2^{|\lambda|}=2^{|\lambda|}\left\|\theta_{\lambda}\right\|_{\mathrm{L}^{2}(I)}=2^{|\lambda|}\left\|\left(\mathrm{Id}-Q_{|\lambda|-1}\right) \theta_{\lambda}\right\|_{\mathrm{L}^{2}(I)} \lesssim\left\|\theta_{\lambda}\right\|_{\mathrm{H}^{1}(I)} .
$$

Here, for $m \in \mathbb{N}_{0}, Q_{m}$ denotes the $\left(\Theta, \Theta^{\prime}\right)$ biorthogonal projector onto $\operatorname{span}\left\{\theta_{\lambda}\right.$ : $|\lambda| \leq m\}$. Using (t1) and (t3), we infer that

$$
\left\|\theta_{\lambda}\right\|_{\mathrm{H}^{1}(I)}^{-1}\left|\int_{I} \theta_{\lambda}^{\prime} \theta_{\mu} \mathrm{d} t\right| \lesssim 2^{-|\lambda|} 2^{-\max (|\lambda|,|\mu|)} 2^{|\lambda|\left(\frac{1}{2}+1\right)} 2^{\frac{1}{2}|\mu|}=2^{-\frac{1}{2}|| \lambda|-| \mu||} .
$$

Finally, we note that any entry of $\left([\Theta]_{\mathrm{H}^{1}(I)}^{\prime}, \Theta\right)_{\mathrm{L}^{2}(I)}$ can be evaluated in closed form in $\mathcal{O}(1)$ work and memory. Schur's Lemma (cf. [19], p. 6, Par. 2) now implies that $\left([\Theta]_{\mathrm{H}^{1}(I)}^{\prime}, \Theta\right)_{\mathrm{L}^{2}(I)}$ and $\left(\Theta,[\Theta]_{\mathrm{H}^{1}(I)}^{\prime}\right)_{\mathrm{L}^{2}(I)}$ are $\infty$-computable.

Note that from $\operatorname{diam}\left(\operatorname{supp} \theta_{\lambda}\right) \lesssim 2^{-|\lambda|}$ (cf. (t1)) and from and (t3) we deduce that $\left\|\theta_{\lambda}\right\|_{\mathrm{H}^{1}(I)} \lesssim 2^{|\lambda|}$, and thus that

$$
\left\|\theta_{\lambda}\right\|_{\mathrm{H}^{1}(I)} \approx 2^{|\lambda|}
$$

Remark 9.2 Suppose that, instead of (t3), the $\theta_{\lambda}$ belong also to $\mathrm{C}^{r_{t}}(I)$ for some $r_{t} \in \mathbb{N}$ (necessarily with $\left.r_{t} \leq d_{t}-2\right)$, i.e., that $\left\|\theta_{\lambda}\right\| \mathrm{W}^{s, \infty}(0,1) \lesssim 2^{|\lambda|\left(\frac{1}{2}+s\right)}$ for $s \in\left\{0, r_{t}+1\right\}$. Then, by subtracting a suitable polynomial of degree $r_{t}$ from $\theta_{\lambda}^{\prime}$ in (9.4), and using that $\theta_{\mu}$ has $\tilde{d}_{t} \geq d_{t} \geq r_{t}$ vanishing moments one deduces that, for $|\lambda| \leq|\mu|$,

$$
\left\|\theta_{\lambda}\right\|_{\mathrm{H}^{1}(I)}^{-1}\left|\int_{I} \theta_{\lambda}^{\prime} \theta_{\mu} \mathrm{d} t\right| \lesssim 2^{-|| \lambda|-| \mu||\left(\frac{1}{2}+r_{t}\right)}
$$


Similarly, for $|\lambda| \geq|\mu|$, using integration by parts one obtains that

$$
\left\|\theta_{\lambda}\right\|_{\mathrm{H}^{1}(I)}^{-1}\left|\int_{I} \theta_{\lambda}^{\prime} \theta_{\mu} \mathrm{d} t\right| \lesssim 2^{-|| \lambda|-| \mu||\left(\frac{3}{2}+r_{t}\right)}
$$

if $t \mapsto \theta_{\lambda}(t) \theta_{\mu}(t)$ vanishes on $\partial I$. Since the wavelets in time do not satisfy a homogeneous Dirichlet boundary condition, there are $\lambda, \mu \in \nabla_{t}$ with $|\lambda| \geq|\mu|$ for which $t \mapsto \theta_{\lambda}(t) \theta_{\mu}(t)$ does not vanish at the boundary. For such entries (9.4) cannot be improved.

Remark 9.3 In reference [6], multiresolutions $\Theta=\left(\theta_{\lambda}\right)_{\lambda \in \nabla_{t}}$ were constructed for which the matrices $\left([\Theta]_{\mathrm{H}^{1}(I)}^{\prime}, \Theta\right)_{\mathrm{L}^{2}(I)}$ are sparse without compression and which satisfy a homogeneous Dirichlet boundary condition at $t=T$.

$9.3 s^{*}$-computability of $(\Upsilon, \Upsilon)_{\mathcal{H}}$ and $\left([\Upsilon] \mathcal{V}^{*},[\Upsilon] \mathcal{V}\right)_{\mathcal{H}}$ and of its adjoint

In view of the $\mathrm{L}^{2}(H, \mu)$-orthonormality (3.5) of the collection $\Upsilon$ of Hermite polyno-

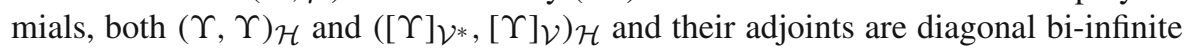
matrices that are therefore $\infty$-computable.

$9.4 s^{*}$-computability of $\int_{I} \mathfrak{a}(\Theta \otimes[\Upsilon] \mathcal{V}, \Theta \otimes[\Upsilon] \mathcal{V}) \mathrm{d} t$ and of its adjoint

We have that

$$
\int_{I} \mathfrak{a}(\Theta \otimes[\Upsilon] \mathcal{V}, \Theta \otimes[\Upsilon] \mathcal{V}) \mathrm{d} t=(\Theta, \Theta)_{\mathrm{L}^{2}(I)} \otimes \mathfrak{a}([\Upsilon] \mathcal{V},[\Upsilon] \mathcal{V})
$$

Since the form $\mathfrak{a}(\cdot, \cdot)$ defined in (4.7) is symmetric, by Proposition 9.1 it suffices to investigate $s^{*}$-computability of each of the two factors on the right-hand side of (9.5) in order to deduce $s^{*}$-computability of $\mathbf{B}$ and of $\mathbf{B}^{*}$.

As was noted in [20, Sec. 8.5], under the assumptions (t1)-(t4) above, $(\Theta, \Theta)_{\mathrm{L}^{2}(I)}$ is $\infty$-computable, so it remains to address the $s^{*}$-computability of $\mathfrak{a}([\Upsilon] \mathcal{V},[\Upsilon] \mathcal{V})$.

In view of the definition (4.7) of $\mathfrak{a}(\cdot, \cdot)$ in (4.6) and of Definition 7.8, $s^{*}$ computability of the bi-infinite matrix $\mathbf{G}=\mathfrak{a}([\Upsilon] \mathcal{V},[\Upsilon] \mathcal{V})$ depends on the structure of the bi-infinite matrix $\mathbf{A}=\left(A_{i j}\right)_{i, j=1}^{\infty}$ in (4.6). Thanks to (9.5),

$$
\mathfrak{a}([\Upsilon] \mathcal{V},[\Upsilon] \mathcal{V})=\left(\mathbf{G}_{\gamma, \gamma^{\prime}}\right)_{\gamma, \gamma \in \Gamma},
$$


where the entries $G_{\gamma \gamma^{\prime}}$ are, for $\gamma, \gamma^{\prime} \in \Gamma$, evaluated (assuming $d=1$ for ease of notation and using (9.3)) as follows:

$$
\begin{aligned}
G_{\gamma \gamma^{\prime}} & =\sum_{i, j \geq 1} A_{i j}\left(D_{\gamma}^{\lambda}\right)^{-1}\left(\mathrm{D}_{q_{i}} H_{\gamma}, \mathrm{D}_{q_{j}} H_{\gamma^{\prime}}\right)_{\mathrm{L}^{2}(D, \mu)}\left(D_{\gamma^{\prime}}^{\lambda}\right)^{-1} \\
& =\sum_{i, j \geq 1} A_{i j}\left(D_{\gamma}^{\lambda}\right)^{-1}\left(\sqrt{\gamma_{i} / \lambda_{i}} H_{\gamma_{i}-1} H_{\gamma}^{(i)}, \sqrt{\gamma_{j}^{\prime} / \lambda_{j}} H_{\gamma_{j}^{\prime}-1} H_{\gamma^{\prime}}^{(j)}\right)_{\mathrm{L}^{2}(D, \mu)}\left(D_{\gamma^{\prime}}^{\lambda}\right)^{-1} \\
& =\left(D_{\gamma}^{\lambda}\right)^{-1}\left(D_{\gamma^{\prime}}^{\lambda}\right)^{-1} \sum_{i, j \geq 1} A_{i j} \sqrt{\frac{\gamma_{i} \gamma_{j}^{\prime}}{\lambda_{i} \lambda_{j}}}\left(H_{\gamma_{i}-1} H_{\gamma^{(i)}}^{(i)} H_{\gamma_{j}^{\prime}-1} H_{\gamma^{\prime}}^{(j)}\right)_{\mathrm{L}^{2}(D, \mu)}
\end{aligned}
$$

To verify $s^{*}$-admissibility of the matrix $\mathbf{G}$, we impose additional hypotheses on the matrix A. We consider two cases: (i) $\mathbf{A}$ is diagonal; and (ii) $\mathbf{A}$ is tridiagonal.

First, consider the case $A_{i j}=\delta_{i j}$. Inserting this into (9.6), we obtain from the $\mathrm{L}^{2}(D, \mu)$ orthonormality (3.5) of the collection $\left\{H_{\gamma}: \gamma \in \Gamma\right\}$ that

$$
\begin{aligned}
G_{\gamma \gamma^{\prime}} & =\left(D_{\gamma}^{\lambda}\right)^{-1}\left(D_{\gamma^{\prime}}^{\lambda}\right)^{-1} \sum_{i, j \geq 1} \delta_{i j} \sqrt{\frac{\gamma_{i} \gamma_{j}^{\prime}}{\lambda_{i} \lambda_{j}}}\left(H_{\gamma_{i}-1} H_{\gamma}^{(i)}, H_{\gamma_{j}^{\prime}-1} H_{\gamma^{\prime}}^{(j)}\right)_{\mathrm{L}^{2}(D, \mu)} \\
& =\left(D_{\gamma}^{\lambda}\right)^{-1}\left(D_{\gamma^{\prime}}^{\lambda}\right)^{-1} \sum_{i \geq 1} \sqrt{\frac{\gamma_{i} \gamma_{i}^{\prime}}{\lambda_{i}^{2}}}\left(H_{\gamma_{i}-1} H_{\gamma}^{(i)}, H_{\gamma_{i}^{\prime}-1} H_{\gamma^{\prime}}^{(i)}\right)_{\mathrm{L}^{2}(D, \mu)} \\
& =\delta_{\gamma, \gamma^{\prime}}\left(D_{\gamma}^{\lambda}\right)^{-1}\left(D_{\gamma^{\prime}}^{\lambda}\right)^{-1} \sum_{i \geq 1} \sqrt{\frac{\gamma_{i} \gamma_{i}^{\prime}}{\lambda_{i}^{2}}} \\
& =\delta_{\gamma, \gamma^{\prime}} .
\end{aligned}
$$

For $A_{i j}=\delta_{i j}$ the bi-infinite matrix $\mathbf{G}$ is diagonal and, therefore, $\infty$-computable.

We next turn to the tridiagonal matrices A presented in Example 4.1. Here,

$$
A_{i j}=\delta_{i j}+\epsilon_{i} \delta_{i, j-1}+\epsilon_{i} \delta_{i, j+1}, \quad i, j=1,2, \ldots
$$

The diagonal term having been already discussed, by superposition we may confine ourselves to investigating the computability of $\mathbf{G}$ for $A_{i j}=\epsilon_{i} \delta_{i, j \pm 1}, i, j=1,2, \ldots$. Given $\gamma, \gamma^{\prime} \in \Gamma$, we calculate, as before, that

$$
\begin{aligned}
G_{\gamma \gamma^{\prime}} & =\left(D_{\gamma}^{\lambda}\right)^{-1}\left(D_{\gamma^{\prime}}^{\lambda}\right)^{-1} \sum_{i, j \geq 1} \epsilon_{i} \delta_{i, j \pm 1} \sqrt{\frac{\gamma_{i} \gamma_{j}^{\prime}}{\lambda_{i} \lambda_{j}}}\left(H_{\gamma_{i}-1} H_{\gamma}^{(i)}, H_{\gamma_{j}^{\prime}-1} H_{\gamma^{\prime}}^{(j)}\right)_{\mathrm{L}^{2}(D, \mu)} \\
& =\left(D_{\gamma}^{\lambda}\right)^{-1}\left(D_{\gamma^{\prime}}^{\lambda}\right)^{-1} \sum_{i \geq 1} \epsilon_{i} \sqrt{\frac{\gamma_{i} \gamma_{i \mp 1}^{\prime}}{\lambda_{i} \lambda_{i \mp 1}}}\left(H_{\gamma_{i}-1} H_{\gamma}^{(i)}, H_{\gamma_{i \mp 1}^{\prime}-1} H_{\gamma^{\prime}}^{(i \mp 1)}\right)_{\mathrm{L}^{2}(D, \mu)}
\end{aligned}
$$

We evaluate

$$
\left(H_{\gamma_{i}-1} H_{\gamma}^{(i)}, H_{\gamma_{i \mp 1}^{\prime}-1} H_{\gamma^{\prime}}^{(i \mp 1)}\right)_{\mathrm{L}^{2}(D, \mu)}=\left\{\begin{array}{l}
1 \text { if } \gamma_{i}^{\prime}=\gamma_{i}-1 \wedge \gamma_{i \mp 1}^{\prime}=\gamma_{i \mp 1}+1 \wedge \gamma_{j}^{\prime}=\gamma_{j} \\
j \in\{i, i \mp 1\}^{c} \\
0 \text { otherwise. }
\end{array}\right.
$$


For $\gamma \in \Gamma$ let us define the support of $\gamma$ by $\operatorname{supp}(\gamma):=\left\{i \in \mathbb{N}: \gamma_{i} \neq 0\right\}$. Then, $G_{\gamma \gamma^{\prime}}=0$ if $\operatorname{supp}(\gamma) \cap \operatorname{supp}\left(\gamma^{\prime}\right)=\emptyset$. Therefore $\mathbf{G}$ is sparse. If, on the other hand, $\operatorname{supp}(\gamma) \cap \operatorname{supp}\left(\gamma^{\prime}\right)$ is nonempty, then, for each $\gamma \in \Gamma$ and each $i \in \mathbb{N}$, the expression

$$
\left(H_{\gamma_{i}-1} H_{\gamma}^{(i)}, H_{\gamma_{i \mp 1}^{\prime}-1} H_{\gamma^{\prime}}^{(i \mp 1)}\right)_{\mathrm{L}^{2}(D, \mu)}
$$

is equal to 1 for exactly one $\gamma^{\prime}(\gamma, i) \in \Gamma$. From the above calculations we observe that for this column index $\gamma^{\prime}(\gamma, i)$, we have that $G_{\gamma, \gamma^{\prime}(\gamma, i)}=\epsilon_{i}$; we thus deduce that for the Fokker-Planck equation (2.17) with tridiagonal coefficient matrices $\mathbf{A}$ as in Example 4.1, the bi-infinite matrix $\mathbf{G}$ is still highly sparse: it contains in each row with index $\gamma \in \Gamma$, for each $\epsilon_{i}$ with $i \in \operatorname{supp}(\gamma)$, exactly one off-diagonal entry $\epsilon_{i}$ in the column with index $\gamma^{\prime}(\gamma, i)$.

We now refer to Definition 7.8 and verify condition (7.3): for $\bar{s}>0$, we have

$$
c_{\mathbf{G}, \bar{s}}:=\sup _{N \in \mathbb{N}} N\left\|\mathbf{G}-\mathbf{G}^{[N]}\right\|_{\ell^{2}(\Gamma) \rightarrow \ell^{2}(\Gamma)}^{1 / \bar{s}}<\infty .
$$

Since

$$
\left\|\mathbf{G}-\mathbf{G}^{[N]}\right\|_{\ell^{2}(\Gamma) \rightarrow \ell^{2}(\Gamma)}^{2} \leq \sup _{\gamma \in \Gamma} \sum_{\gamma^{\prime} \in \Gamma}\left|G_{\gamma \gamma^{\prime}}-G_{\gamma \gamma^{\prime}}^{[N]}\right|^{2},
$$

we define $\mathbf{G}^{[N]}$ "row"-wise for $N \in \mathbb{N}$ as follows: if $N=1$, we select $\mathbf{G}^{[N]}$ to be the diagonal part of $\mathbf{G}$. If $N>1$, we define $\mathbf{G}^{[N]}$ to contain, in the off-diagonal of the "row" associated with index $\gamma \in \Gamma$, at most $N$ nonzero elements $G_{\gamma \gamma}$ ' where $\gamma^{\prime}=\gamma^{\prime}(\gamma, i)$ with the index $i$ such that $i \in\left\{j: \gamma_{j} \neq 0\right\} \cap\left\{j: \epsilon_{j}^{[N-1]} \neq 0\right\}$. Here, for a given sequence $\epsilon \in \ell^{2}(\mathbb{N})$ in the definition (4.3) of the infinite, tridiagonal matrix $\mathbf{A}$ appearing in the bilinear form $\mathfrak{a}(\cdot, \cdot)$, we denote by $\epsilon^{[N]}$ its best $(N-1)$-term approximation in $\ell^{2}(\mathbb{N})$. Then, for all $N \in \mathbb{N}$ and for every $0<p \leq 2$,

$$
\left\|\epsilon-\epsilon^{[N]}\right\|_{\ell^{2}(\mathbb{N})} \leq N^{-(1 / p-1 / 2)}\|\epsilon\|_{\ell^{p}(\mathbb{N})}
$$

by Stechkin's Lemma ${ }^{1}$. It therefore follows from the definition (9.7) of the entries $G_{\gamma \gamma^{\prime}}$ of $\mathbf{G}$ and from the above calculations that

$\forall N \in \mathbb{N}: \quad\left\|\mathbf{G}-\mathbf{G}^{[N]}\right\|_{\ell^{2}(\Gamma) \rightarrow \ell^{2}(\Gamma)} \leq\left\|\epsilon-\epsilon^{[N-1]}\right\|_{\ell^{2}(\mathbb{N})} \leq 2^{1 / p-1 / 2} N^{-(1 / p-1 / 2)}\|\epsilon\|_{\ell^{p}(\mathbb{N})}$, from which we deduce, with $C_{p}:=2^{(1 / p-1 / 2) / \bar{s}}$, that

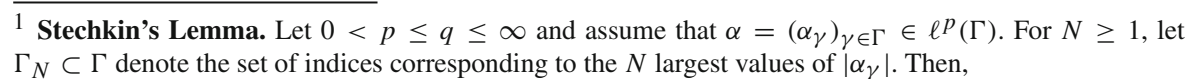
$\left(\sum_{\gamma \notin \Gamma_{N}}\left|\alpha_{\gamma}\right|^{q}\right)^{1 / q} \leq N^{-r}\|\alpha\|_{\ell p}(\Gamma), \quad$ where $r:=\frac{1}{p}-\frac{1}{q} \geq 0$.

The proof is elementary; see, for example, [11]. 


$$
\begin{aligned}
c_{\mathbf{G}, \bar{s}} & =\sup _{N \in \mathbb{N}} N\left\|\mathbf{G}-\mathbf{G}^{[N]}\right\|_{\ell^{2}(\Gamma)}^{1 / \bar{s}} \leq C_{p}\left(\sup _{N \in \mathbb{N}} N^{1-(1 / p-1 / 2) / \bar{s}}\right)\|\epsilon\|_{\ell p(\mathbb{N})}^{1 / \bar{s}} \\
& =2\|\epsilon\|_{\ell^{p}(\mathbb{N})}^{1 / \bar{s}}<\infty
\end{aligned}
$$

provided that: $\epsilon \in \ell^{p}(\mathbb{N})$ with $0<p<2$ and $\bar{s}=\bar{s}(p)$ is chosen as

$$
0<\bar{s}(p):=1 / p-1 / 2
$$

Referring to the definition of $s^{*}$-computability (cf. Definition 7.8), we infer that $\mathbf{G}$ is $s^{*}$-computable with any $0<s^{*} \leq \bar{s}(p)$ if the sequence $\epsilon$ in Example 4.1 belongs to $\ell^{p}(\mathbb{N})$ with some $0<p<2$, resp. with $s^{*}=1 / p-1 / 2$ (this encompasses the previous case, if $p=0$ is understood to indicate that $\epsilon$ is the zero sequence).

\section{Equations with drift}

\subsection{Bounded invertibility of $B$ and $B^{*}$ for nonsymmetric $\mathfrak{a}(\cdot, \cdot)$}

So far, we have assumed that the "spatial" differential operator is defined by the symmetric bilinear form $\mathfrak{a}(\cdot, \cdot)$. This is always possible when the vector function appearing in the coefficient of the drift term in the Fokker-Planck equation is the gradient of a potential, by introducing Maxwellian weighted spaces $\mathrm{L}_{M}^{2}(D)$ and $\mathrm{H}_{M}^{1}(D)$ and by rescaling the probability density function by the Maxwellian; see, for example, [1-3]. In cases when the drift term cannot be removed by transformation to Maxwellianweighted operators, however, nonsymmetric bilinear forms $\mathfrak{a}(\cdot, \cdot)$ must be considered. The bounded invertibility of the operator $B$ corresponding to the bilinear form $\mathfrak{B}^{*}(\cdot, \cdot)$ in (4.6) must then be considered separately.

We now address the $s^{*}$-computability of the drift term. Let $\mu$ be the countable product of Gaussian measures $\mu_{k}, k \geq 1$, on $\mathbb{R}^{d}$ with trace-class covariance operator $Q$. The new contribution added to the symmetric bilinear form $\mathfrak{a}(\cdot, \cdot)$ that was under consideration in previous sections is assumed, for a sequence $\sigma=\left(\sigma_{k}\right)_{k \geq 1} \in \ell^{\infty}\left(\mathbb{N} ; \mathbb{R}^{d \times d}\right)$, the space of bounded sequences of $d \times d$ matrices, to be the bilinear form $\mathfrak{d}(\cdot, \cdot)$ defined by

$$
\mathfrak{d}(\widehat{\psi}, \widehat{\varphi})=-\sum_{k \geq 1}\left(\sigma_{k} q_{k} \widehat{\psi}, \mathrm{D}_{k} \widehat{\varphi}\right)_{\mathrm{L}^{2}(H, \mu)}
$$

The choice of (10.1) is motivated by Fokker-Planck equations that arise in the context of mesoscopic bead-spring chain models for the viscous, incompressible flow of dilute polymers in $d$-dimensional domains with $d \in\{2,3\}$, with chains consisting of $K+1$ beads that are linearly connected with $K$ Hookean springs. The kinematics of these springs are statistically described by a configuration vector $q=\left(q_{1}^{\top}, \ldots, q_{K}^{\top}\right)^{\top} \in$ $\mathbb{R}^{K d}$ with $q_{k} \in \mathbb{R}^{d}$ and $K \gg 1$. The probability density function that is sought as the solution of the associated Fokker-Planck equation is then a function of $K d$ spatial variables with $K \gg 1$ and time $t$, and for each $k=1, \ldots, K, \sigma_{k} \in \mathbb{R}^{d \times d}$ is a constant matrix with respect to $q=\left(q_{1}^{\top}, \ldots, q_{K}^{\top}\right)^{\top}$; see, for example, [1-3]. 
Proposition 10.1 Assume that $\sigma \in \ell^{\infty}\left(\mathbb{N} ; \mathbb{R}^{d \times d}\right)$ and that the covariance operator $Q$ of the Gaussian measure $\mu$ on $H$ is trace-class. Then, $\mathfrak{d}(\cdot, \cdot): \mathrm{W}^{1,2}(H, \mu) \times$ $\mathrm{W}^{1,2}(H, \mu) \rightarrow \mathbb{R}$ is continuous and

$$
\begin{aligned}
|\mathfrak{d}(\widehat{\psi}, \widehat{\varphi})| \leq & \|\sigma\|_{\ell^{\infty}\left(\mathbb{N} ; \mathbb{R}^{d \times d}\right)}\left(2 \operatorname{Tr} Q \int_{H}|\widehat{\psi}(q)|^{2} \mu(\mathrm{d} q)\right. \\
& \left.+4\|Q\|^{2} \int_{H}|\mathrm{D} \widehat{\psi}(q)|^{2} \mu(\mathrm{d} q)\right)^{1 / 2}\|\mathrm{D} \widehat{\varphi}\|_{\mathrm{L}^{2}(H, \mu)}
\end{aligned}
$$

Proof We write

$$
\begin{aligned}
|\mathfrak{d}(\widehat{\psi}, \widehat{\varphi})| & \leq\|\sigma\|_{\ell^{\infty}\left(\mathbb{N} ; \mathbb{R}^{d \times d}\right)}\left(\sum_{k \geq 1}\left\|\left|q_{k}\right| \widehat{\psi}\right\|_{\mathrm{L}^{2}(H, \mu)}^{2}\right)^{1 / 2}\left(\sum_{k^{\prime} \geq 1}\left\|\mathrm{D}_{k^{\prime}} \widehat{\varphi}\right\|_{\mathrm{L}^{2}(H, \mu)}^{2}\right)^{1 / 2} \\
& =\|\sigma\|_{\ell^{\infty}\left(\mathbb{N} ; \mathbb{R}^{d \times d}\right)}\left(\sum_{k \geq 1}\left\|\left|q_{k}\right| \widehat{\psi}\right\|_{\mathrm{L}^{2}(H, \mu)}^{2}\right)^{1 / 2}\|\mathrm{D} \widehat{\varphi}\|_{\mathrm{L}^{2}(H, \mu)} \cdot
\end{aligned}
$$

By noting [10, Prop. 9.2.10] and the assumption that $\widehat{\psi} \in \mathrm{W}^{1,2}(H, \mu)$, we deduce that, for a Gaussian measure $\mu$ with trace-class covariance $Q$,

$$
\int_{H}\|q\|^{2}|\widehat{\psi}(q)|^{2} \mu(\mathrm{d} q) \leq 2 \operatorname{Tr} Q \int_{H}|\widehat{\psi}(q)|^{2} \mu(\mathrm{d} q)+4\|Q\|^{2} \int_{H}|\mathrm{D} \widehat{\psi}(q)|^{2} \mu(\mathrm{d} q) .
$$

This then yields the desired inequality (10.2).

The bound (10.2) implies a Garding inequality for the Fokker-Planck operator with drift.

Proposition 10.2 Assume that the covariance operator $Q$ of the Gaussian measure $\mu$ is trace-class, and that the infinite coefficient matrix A satisfies (4.1), (4.2).

Then, the bilinear form

$$
\mathfrak{a}(\cdot, \cdot)+\mathfrak{d}(\cdot, \cdot): \mathcal{V} \times \mathcal{V} \rightarrow \mathbb{R}
$$

is continuous and satisfies the Garding inequality (2.22) in the triple $\mathcal{V} \subset \mathcal{H} \simeq$ $\mathcal{H}^{*} \subset \mathcal{V}^{*}$. In particular, the space-time variational formulation (4.5) with the spatial bilinear form $\mathfrak{a}(\cdot, \cdot)+\mathfrak{d}(\cdot, \cdot)$ in place of $\mathfrak{a}(\cdot, \cdot)$ in (4.6) is well-posed and its bilinear form $\mathfrak{B}^{*}(\cdot, \cdot)$ induces a boundedly invertible operator $B^{*} \in \mathcal{L}\left(\mathcal{Y},\left(\mathcal{X}_{0,\{T\}}\right)^{*}\right)$.

Proof The continuity of the form is evident from the previous proposition and the continuity of $\mathfrak{a}(\cdot, \cdot)$. The Garding inequality follows from the coercivity of $\mathfrak{a}(\cdot, \cdot)$ on $\mathcal{V} \times \mathcal{V}$ and from the inequality (10.2) using a Cauchy inequality with $\varepsilon>0$ sufficiently small. The bounded invertibility of $B^{*} \in \mathcal{L}\left(\mathcal{Y},\left(\mathcal{X}_{0,\{T\}}\right)^{*}\right)$ follows from (2.22) and from Theorem 2.2. 
$10.2 s^{*}$-computability of $\int_{I} \mathfrak{d}(\Theta \otimes[\Upsilon] \mathcal{V}, \Theta \otimes[\Upsilon] \mathcal{V}) \mathrm{d} t$ and of its adjoint

As in Section 9.4 thanks to the independence of the sequence $\sigma \in \ell^{\infty}\left(\mathbb{N} ; \mathbb{R}^{d \times d}\right)$ of $t$, we have that

$$
\int_{I} \mathfrak{d}(\Theta \otimes[\Upsilon] \mathcal{V}, \Theta \otimes[\Upsilon] \mathcal{V}) \mathrm{d} t=(\Theta, \Theta)_{\mathrm{L}^{2}(I)} \otimes \mathfrak{d}([\Upsilon] \mathcal{V},[\Upsilon] \mathcal{V})
$$

As in the discussion of (9.5), the sparsity of the factor $(\Theta, \Theta)_{\mathrm{L}^{2}(I)}$ is considered in $\left[20\right.$, Sec. 8.5]. Sparsity and $s^{*}$-computability of $\mathfrak{d}(\cdot, \cdot)$ are therefore determined by that of the infinite drift matrix $\mathbf{D}=\mathbf{D}[\sigma]$ defined via $\mathfrak{d}([\Upsilon] \mathcal{V},[\Upsilon] \mathcal{V})=\left(D_{\gamma, \gamma^{\prime}}\right)_{\gamma, \gamma \in \Gamma}$. With the Hermite polynomial Riesz basis $[\Upsilon] \mathcal{V}$ of $\mathrm{L}^{2}(H, \mu)$, the matrix entries $D_{\gamma \gamma^{\prime}}=$ $D_{\gamma \gamma^{\prime}}[\sigma]$ are (assuming, once again, for the sake of simplicity of the exposition that $d=1$ and denoting $\ell^{\infty}\left(\mathbb{N} ; \mathbb{R}^{d \times d}\right)$ by $\ell^{\infty}$; abbreviating $\mathrm{D}_{q_{k}}$ as $\mathrm{D}_{k}$ to simplify the notation; and recalling the definition (9.1) of $D_{\gamma}^{\lambda}$ ):

$$
D_{\gamma \gamma^{\prime}}[\sigma]=\sum_{k \geq 1} \sigma_{k}\left(D_{\gamma}^{\lambda}\right)^{-1}\left(D_{\gamma^{\prime}}^{\lambda}\right)^{-1}\left(\mathrm{D}_{k}\left(q_{k} H_{\gamma}\right), H_{\gamma^{\prime}}\right)_{\mathrm{L}^{2}(H, \mu)}
$$

For the ensuing calculations, we define

$$
\theta_{\gamma \gamma^{\prime}}(k):=\left(\mathrm{D}_{k}\left(q_{k} H_{\gamma}\right), H_{\gamma^{\prime}}\right)_{\mathrm{L}^{2}(H, \mu)}
$$

and observe that

$$
\begin{aligned}
\theta_{\gamma \gamma^{\prime}}(k) & =\left(H_{\gamma}, H_{\gamma^{\prime}}\right)_{\mathrm{L}^{2}(H, \mu)}+\left(q_{k} \mathrm{D}_{k} H_{\gamma}, H_{\gamma^{\prime}}\right)_{\mathrm{L}^{2}(H, \mu)} \\
& =\delta_{\gamma \gamma^{\prime}}+\left(q_{k} \mathrm{D}_{k} H_{\gamma}, H_{\gamma^{\prime}}\right)_{\mathrm{L}^{2}(H, \mu)} .
\end{aligned}
$$

In order to calculate the second term on the right-hand side of (10.4), we note that, by $(9.3)$,

$$
\mathrm{D}_{k} H_{\gamma}(q)=\left\{\begin{array}{cl}
\sqrt{\frac{\gamma_{k}}{\lambda_{k}}} H_{\gamma-e_{k}}(q) & \text { if } \gamma_{k} \geq 1 \\
0 & \text { otherwise }
\end{array}\right.
$$

where $e_{k} \in \Gamma$ denotes the multi-index with entry 1 in position $k$ and with zero entries in all other positions. Also, thanks to the three-term recurrence relation

$$
q_{k} H_{\gamma_{k}}\left(q_{k}\right)=\sqrt{\gamma_{k}+1} H_{\gamma_{k}+1}\left(q_{k}\right)+\sqrt{\gamma_{k}} H_{\gamma_{k}-1}\left(q_{k}\right), \quad \gamma_{k} \in \mathbb{N}_{0},
$$

for the univariate polynomials (3.4) (see, (9.1.3) in [10]), with the notational convention that $H_{m}\left(q_{k}\right) \equiv 0$ when $m<0$, we have that 


$$
\begin{aligned}
q_{k} \mathrm{D}_{k}\left[H_{\gamma_{k}}\left(\frac{q_{k}}{\sqrt{\lambda_{k}}}\right)\right]= & q_{k} \sqrt{\frac{\gamma_{k}}{\lambda_{k}}} H_{\gamma_{k}-1}\left(\frac{q_{k}}{\sqrt{\lambda_{k}}}\right)=\frac{\gamma_{k}}{\sqrt{\lambda_{k}}} H_{\gamma_{k}}\left(\frac{q_{k}}{\sqrt{\lambda_{k}}}\right) \\
& +\sqrt{\frac{\gamma_{k}\left(\gamma_{k}-1\right)}{\lambda_{k}}} H_{\gamma_{k}-2}\left(\frac{q_{k}}{\sqrt{\lambda_{k}}}\right) .
\end{aligned}
$$

Consequently, upon multiplying both sides of the last identity with $H_{\gamma}^{(k)}$, we deduce that

$$
q_{k} \mathrm{D}_{k} H_{\gamma}=\frac{\gamma_{k}}{\sqrt{\lambda_{k}}} H_{\gamma}+\sqrt{\frac{\gamma_{k}\left(\gamma_{k}-1\right)}{\lambda_{k}}} H_{\gamma-2 e_{k}} \text {, }
$$

which, upon substitution into (10.4) and then inserting the resulting expression into (10.3) yields, for $\gamma, \gamma^{\prime} \in \Gamma$, that

$$
\begin{aligned}
D_{\gamma \gamma^{\prime}}[\sigma]= & \sum_{k \geq 1} \sigma_{k}\left(\left(1+\frac{\gamma_{k}}{\sqrt{\lambda_{k}}}\right) \delta_{\gamma \gamma^{\prime}}+\sqrt{\frac{\gamma_{k}\left(\gamma_{k}-1\right)}{\lambda_{k}}} \delta_{\gamma-2 e_{k}, \gamma^{\prime}}\right) \\
& \times\left\langle\gamma, \lambda^{-1}\right\rangle_{*}^{-1 / 2}\left\langle\gamma^{\prime}, \lambda^{-1}\right\rangle_{*}^{-1 / 2},
\end{aligned}
$$

with the notational convention that $\delta_{\gamma-2 e_{k}, \gamma^{\prime}}=0$ for all $\gamma, \gamma^{\prime} \in \Gamma$ such that $\gamma-2 e_{k} \notin$ $\Gamma$. We observe that $\mathbf{D}[\sigma]$ depends linearly on the sequence $\sigma$. The verification of the $s^{*}$-computability now proceeds analogously as in the case of $\mathbf{A}[\epsilon]$.

We denote by $\sigma^{[N]}$ an $N$-term approximation of the sequence $\sigma$ and observe that $\mathbf{D}^{[N]}[\sigma]:=\mathbf{D}\left[\sigma^{[N]}\right]$ has at most $2 N+1$ nonzero entries in each row with index $\gamma \in \Gamma$. To verify $s^{*}$-computability, by Definition 7.8 we must bound

$$
c_{\mathbf{D}, \bar{s}}=\sup _{N \in \mathbb{N}} N\left\|\mathbf{D}[\sigma]-\mathbf{D}^{[N]}[\sigma]\right\|_{\ell^{2}(\Gamma) \rightarrow \ell^{2}(\Gamma)}^{1 / \bar{s}} \leq \sup _{N \in \mathbb{N}}(2 N+1)\left\|\mathbf{D}\left[\sigma-\sigma^{[N]}\right]\right\|_{\ell^{2}(\Gamma) \rightarrow \ell^{2}(\Gamma)}^{1 / \bar{s}} .
$$

For a given fixed $\gamma \in \Gamma$ and for any $\gamma^{\prime} \in \Gamma$ and any $k \in \mathbb{N}$, we have that

$$
\begin{aligned}
\theta_{\gamma \gamma^{\prime}}(k)= & \left(1+\frac{\gamma_{k}}{\sqrt{\lambda_{k}}}\right) \delta_{\gamma \gamma^{\prime}}+\sqrt{\frac{\gamma_{k}\left(\gamma_{k}-1\right)}{\lambda_{k}}} \delta_{\gamma-2 e_{k}, \gamma^{\prime}} \\
= & \left\{\begin{array}{cl}
1+\frac{\gamma_{k}}{\sqrt{\lambda_{k}}} & \text { if } \gamma^{\prime}=\gamma, \\
\sqrt{\frac{\gamma_{k}\left(\gamma_{k}-1\right)}{\lambda_{k}}} & \text { if } \gamma^{\prime}=\gamma-2 e_{k} \in \Gamma, \\
0 & \text { otherwise. }
\end{array}\right.
\end{aligned}
$$

We note that since $\gamma^{\prime} \in \Gamma$, and therefore all of its components are nonnegative, the second of these instances can only occur when $\gamma_{k} \geq 2$. Therefore, we can bound 


$$
\begin{aligned}
\left\|\mathbf{D}[\sigma]-\mathbf{D}^{[N]}[\sigma]\right\|_{\ell^{2}(\Gamma) \rightarrow \ell^{2}(\Gamma)}^{2} & \leq \sup _{\gamma \in \Gamma} \sum_{\gamma^{\prime} \in \Gamma}\left|D_{\gamma \gamma^{\prime}}\left[\sigma-\sigma^{[N]}\right]\right|^{2} \\
& =\sup _{\gamma \in \Gamma} \frac{1}{\left\langle\gamma, \lambda^{-1}\right\rangle_{*}} \sum_{\gamma^{\prime} \in \Gamma} \frac{1}{\left\langle\gamma^{\prime}, \lambda^{-1}\right\rangle_{*}}\left|\sum_{k \geq 1}\left(\sigma_{k}-\sigma_{k}^{[N]}\right) \theta_{\gamma \gamma^{\prime}}(k)\right|^{2} .
\end{aligned}
$$

Fixing $\gamma \in \Gamma$ for now, we deduce that

$$
\begin{aligned}
\mathrm{S}_{\gamma} & :=\sum_{\gamma^{\prime} \in \Gamma} \frac{1}{\left\langle\gamma^{\prime}, \lambda^{-1}\right\rangle_{*}}\left|\sum_{k \geq 1}\left(\sigma_{k}-\sigma_{k}^{[N]}\right) \theta_{\gamma \gamma^{\prime}}(k)\right|^{2} \\
& \leq\left\|\sigma-\sigma^{[N]}\right\|_{\ell^{1}(\mathbb{N})}^{2}\left\{\sup _{k \geq 1} \frac{\left(1+\frac{\gamma_{k}}{\sqrt{\lambda_{k}}}\right)^{2}}{\left\langle\gamma, \lambda^{-1}\right\rangle_{*}}+\sup _{k \geq 1: \gamma_{k} \geq 2} \frac{\frac{\gamma_{k}\left(\gamma_{k}-1\right)}{\lambda_{k}}}{\left\langle\gamma-2 e_{k}, \lambda^{-1}\right\rangle_{*}}\right\} .
\end{aligned}
$$

This implies that

$$
\begin{aligned}
& \left\|\mathbf{D}-\mathbf{D}^{[N]}\right\|_{\ell^{2}(\Gamma) \rightarrow \ell^{2}(\Gamma)}^{2} \leq \sup _{\gamma \in \Gamma} \frac{\mathrm{S}_{\gamma}}{\left\langle\gamma, \lambda^{-1}\right\rangle_{*}} \\
& \quad \leq \sup _{\gamma \in \Gamma}\left\{\sup _{k \geq 1} \frac{\left(1+\frac{\gamma_{k}}{\sqrt{\lambda_{k}}}\right)^{2}}{\left\langle\gamma, \lambda^{-1}\right\rangle_{*}^{2}}+\sup _{k \geq 1: \gamma_{k} \geq 2} \frac{\frac{\gamma_{k}\left(\gamma_{k}-1\right)}{\lambda_{k}}}{\left\langle\gamma, \lambda^{-1}\right\rangle_{*}\left\langle\gamma-2 e_{k}, \lambda^{-1}\right\rangle_{*}}\right\}\left\|\sigma-\sigma^{[N]}\right\|_{\ell^{1}(\mathbb{N})}^{2} .
\end{aligned}
$$

We bound the suprema in the curly brackets as follows. For $\gamma=0$, the first supremum is equal to 1 , while the second supremum is 0 by definition, as it is taken over an empty set. Let us now consider the nontrivial case, when $\gamma \in \Gamma \backslash\{0\}$. Since $|\gamma|<\infty$, the "support-set" of this index sequence $\gamma, S(\gamma):=\left\{j \in \mathbb{N}: \gamma_{j} \neq 0\right\}$, is finite. To bound the first term, we note that the finiteness of $S(\gamma)$ implies that there exists $k^{*} \in N$ such that $\sup _{k} \gamma_{k} / \sqrt{\lambda_{k}}=\max _{k} \gamma_{k} / \sqrt{\lambda_{k}}=\gamma_{k^{*}} / \sqrt{\lambda_{k^{*}}}=:|\gamma / \sqrt{\lambda}|_{\infty}$, and we may bound

$$
\begin{aligned}
\sup _{k} \frac{\left(1+\frac{\gamma_{k}}{\sqrt{\lambda_{k}}}\right)^{2}}{\left\langle\gamma, \lambda^{-1}\right\rangle_{*}^{2}} & \leq 2\left(1+\left|\frac{\gamma}{\sqrt{\lambda}}\right|_{\infty}^{2}\right)\left(\sum_{j \geq 1} \frac{\gamma_{j}}{\lambda_{j}}\right)^{-2}=2\left(1+\left|\frac{\gamma}{\sqrt{\lambda}}\right|_{\infty}^{2}\right)\left(\sum_{j \in S(\gamma)} \frac{\gamma_{j}}{\lambda_{j}}\right)^{-2} \\
& \leq 2\left(1+\left|\frac{\gamma}{\sqrt{\lambda}}\right|_{\infty}^{2}\right)\left(\frac{1}{\sqrt{\lambda_{k^{*}}}} \frac{\gamma_{k^{*}}}{\sqrt{\lambda_{k^{*}}}}\right)^{-2}=2 \lambda_{k^{*}} \frac{1+|\gamma / \sqrt{\lambda}|_{\infty}^{2}}{|\gamma / \sqrt{\lambda}|_{\infty}^{2}} \\
& =2 \lambda_{k^{*}}\left(\frac{1}{|\gamma / \sqrt{\lambda}|_{\infty}^{2}}+1\right) \leq 2 \lambda_{k^{*}}\left(\frac{1}{|1 / \sqrt{\lambda}|_{\infty}^{2}}+1\right) \leq 2 \lambda_{1}\left(\lambda_{1}+1\right) \\
& \leq 4\left[\max \left(1, \lambda_{1}\right)\right]^{2}=[2 \max (1,\|Q\|)]^{2}<\infty .
\end{aligned}
$$

The second term in the curly brackets is bounded analogously. Indeed, assuming that $\gamma-2 e_{k} \in \Gamma$ (for, else, the supremum in the second term is taken over an empty set and is therefore equal to 0 by definition), we have that 


$$
\begin{aligned}
& \quad \sup _{k \geq 1: \gamma_{k} \geq 2} \frac{\frac{\gamma_{k}\left(\gamma_{k}-1\right)}{\lambda_{k}}}{\left\langle\gamma, \lambda^{-1}\right\rangle_{*}\left\langle\gamma-2 e_{k}, \lambda^{-1}\right\rangle_{*}} \leq \frac{|\gamma / \lambda|_{\infty}}{\left\langle\gamma, \lambda^{-1}\right\rangle_{*}} \sup _{k \geq 1: \gamma_{k} \geq 2} \frac{\left(\gamma_{k}-2\right)+1}{\left\langle\gamma-2 e_{k}, \lambda^{-1}\right\rangle_{*}} \\
& \leq \sup _{k \geq 1: \gamma_{k} \geq 2} \frac{\left(\gamma_{k}-2\right)+1}{\left\langle\gamma-2 e_{k}, \lambda^{-1}\right\rangle_{*}} \leq \sup _{k \geq 1: \gamma_{k} \geq 2} \frac{\lambda_{k} \frac{\gamma_{k}-2}{\lambda_{k}}}{\left\langle\gamma-2 e_{k}, \lambda^{-1}\right\rangle_{*}} \\
& +\sup _{k \geq 1: \gamma_{k} \geq 2} \frac{1}{\left\langle\gamma-2 e_{k}, \lambda^{-1}\right\rangle_{*}} \\
& \leq \lambda_{1}+\max \left(1, \lambda_{1}\right) \leq 2 \max (1,\|Q\|)<\infty .
\end{aligned}
$$

Inserting these bounds into (10.5), we infer that for $\sigma \in \ell^{p}(\mathbb{N})$ with some $0<p<1$ the bi-infinite drift matrix $\mathbf{D}[\sigma]$ is $s^{*}$-computable for any $0<s^{*} \leq 1 / p-1$.

\section{Optimality}

Based on Proposition 9.1 and on the definition (8.7) of the bi-infinite matrix $\mathbf{B}^{*}$, we deduce from these observations our main result.

Theorem 11.1 Consider the space-time variational formulation (4.5) of the infinitedimensional Fokker-Planck equation with bilinear form $\mathfrak{a}(\cdot, \cdot)$ defined in (4.7) corresponding to the tridiagonal matrix $\mathbf{A}$ as in Example 4.1 based on the sequences $\epsilon \in \ell^{p}(\mathbb{N}), \sigma \in \ell^{p}\left(\mathbb{N} ; \mathbb{R}^{d \times d}\right)$ of elements $\epsilon_{i}, \sigma_{i}$ with $0<p<1$. Consider its representation $\mathbf{B}^{*} \mathbf{u}=\mathbf{f}^{*}$ using a temporal wavelet basis $\Theta$ as in section 9.1 and a spatial Hermite polynomial chaos basis $\Upsilon=\left\{H_{\gamma}(q): \gamma \in \Gamma, q \in D\right\}$. Then, for any $\varepsilon>0$, the adaptive wavelet methods from [8] or [7] (and [13]) applied to the normal equation (7.4) with $\mathbf{f}^{*}$ and $\mathbf{B}^{*}$ as in (8.6) and (8.7), respectively, of the bi-infinite matrix representation of the Fokker-Planck equation (2.17) in countably many dimensions produce a Galerkin approximation $\mathbf{u}_{\varepsilon}$ with

$$
\left\|u-\mathbf{u}_{\varepsilon}^{\top}[\Theta \otimes \Upsilon]\right\| \mathcal{Y} \approx\left\|\mathbf{u}-\mathbf{u}_{\varepsilon}\right\| \leq \varepsilon
$$

Suppose that for some $0<s<\min \left\{d_{t}-1, \bar{s}(p)\right\}$ we have that $\mathbf{u} \in \mathcal{A}_{\infty}^{s}\left(\ell^{2}(\nabla \mathcal{Y})\right)$; then,

$$
\operatorname{supp} \mathbf{u}_{\varepsilon} \lesssim \varepsilon^{-1 / s}\|\mathbf{u}\|_{\mathcal{A}_{\infty}^{s}\left(\ell^{2}(\nabla y)\right)}^{1 / s}
$$

The number of arithmetic operations and storage locations required by one call of the space-time adaptive solver with tolerance $\varepsilon$ is bounded by some multiple of $\varepsilon^{-1 / s}\|\mathbf{u}\|_{\mathcal{A}_{\infty}^{s}\left(\ell^{2}\left(\nabla_{\mathcal{Y}}\right)\right)}^{1 / s}+1$.

The above assertions remain valid when in (2.17) the dimension is a finite number $K d$ (where $K$ denotes the chain-length in mesoscopic models of dilute polymers in spatial dimension $d=2,3$ (cf. [1-3] for details), by choosing Gaussian measures $\mu$ with covariance operator $Q$ of finite rank $K$ with the computability and admissibility constants $c_{\mathbf{B}^{*}, s}$ and $a_{\mathbf{B}^{*}, s}$ independent of $K$. 
We remark in closing that for matrices $\mathbf{A}$ as in Example 4.2 this result remains valid, however now with admissibility constants depending on the block $\mathbf{A}_{11}$ in an unspecific way. We also remark that the assumptions on the sequence $\sigma$ could be slightly weakened, as the limit $1 / p-1$ of $s^{*}$-computability of $\mathbf{D}[\sigma]$ is larger than the value $\bar{s}(p)$ found in (9.9).

Acknowledgements The research reported in this paper was carried out at the Hausdorff Institute for Mathematics (HIM), Bonn, during the HIM-Trimester on "High Dimensional Approximation", MayAugust 2011. C.S. was partially supported by the European Research Council (ERC) under the FP7 programme ERC AdG 247277. E.S. was partially supported by the EPSRC Science and Innovation award to the Oxford Centre for Nonlinear PDE (EP/E035027/1).

\section{References}

1. Barrett, J.W., Süli, E.: Existence and equilibration of global weak solutions to kinetic models for dilute polymers I: finitely extensible nonlinear bead-spring chains. Math. Models Methods Appl. Sci. 21(6), 1211-1289 (2011)

2. Barrett, J.W., Süli, E.: Existence and equilibration of global weak solutions to kinetic models for dilute polymers II: Hookean-type bead-spring chains. Math. Models Methods Appl. Sci. 22(5), 1-82 (2012)

3. Barrett, J.W., Süli, E.: Existence of global weak solutions to finitely extensible nonlinear bead-spring chain models for dilute polymers with variable density and viscosity. J. Differ. Equ. 253, 3610-3677 (2012)

4. Bogachev, V., Da Prato, G., Röckner, M.: Existence and uniqueness of solutions for Fokker-Planck equations on Hilbert spaces. J. Evol. Equ. 10(3), 487-509 (2010)

5. Cerrai, S.: Second Order PDE's in Finite and Infinite Dimension, Lecture Notes in Mathematics, MR 1840644 (2002j:35327), vol. 1762, Springer, Berlin (2001)

6. Chegini, N., Stevenson, R.: Adaptive wavelet schemes for parabolic problems: sparse matrices and numerical results. SIAM J. Numer. Anal. 49(1), 182-212 (2011)

7. Cohen, A., Dahmen, W., DeVore, R.: Adaptive wavelet methods for elliptic operator equationsconvergence rates. Math. Comp. 70, 27-75 (2001)

8. Cohen, A., Dahmen, W., DeVore, R.: Adaptive wavelet methods II-beyond the elliptic case. Found. Comput. Math. 2(3), 203-245 (2002)

9. Da Prato, G., Malliavin, P., Nualart, D.: Compact families of Wiener functionals. C. R. Acad. Sci. Paris Sér. I Math. 312(12), 1287-1291 (1992)

10. Da Prato, G., Zabczyk, J.: Second Order Partial Differential Equations in Hilbert Spaces, London Mathematical Society Lecture Note Series, vol. 293. Cambridge University Press, Cambridge (2002)

11. DeVore, R.: Nonlinear approximation. Acta Numer. 7, 51-150 (1998)

12. Dijkema, T.J., Schwab, Ch., Stevenson, R.: An adaptive wavelet method for solving high-dimensional elliptic PDEs. Constr. Approx. 30(3), 423-455 (2009)

13. Gantumur, T., Harbrecht, H., Stevenson, R.: An optimal adaptive wavelet method without coarsening of the iterands. Math. Comput. 76, 615-629 (2007)

14. Griebel, M., Oswald, P.: Tensor product type subspace splittings and multilevel iterative methods for anisotropic problems. Adv. Comput. Math. 4(1-2), 171-206 (1995)

15. Guberović, R., Schwab, Ch., Stevenson, R.: Space-time variational saddle point formulations of Stokes and Navier-Stokes equations. Technical Report, Seminar for Applied Mathematics, ETHZürich, http://www.sam.math.ethz.ch/reports/2011 (Submitted) (2011)

16. Nitsche, P.-A.: Best $N$ term approximation spaces for tensor product wavelet bases. Constr. Approx. 24(1), 49-70 (2006)

17. Peszat, S.: On a Sobolev space of functions of infinite number of variables. Bull. Polish Acad. Sci. Math. 41(1), 55-60 (1993)

18. Pietsch, A.: Nuclear Locally Convex Spaces, Springer, New York, Translated from the second German edition by William H. Ruckle, Ergebnisse der Mathematik und ihrer Grenzgebiete, Band 66 (1972) 
19. Schur, I.: Bemerkungen zur Theorie der Beschränkten Bilinearformen mit unendlich vielen Veränderlichen. J. Reine Angew. Math. 140, 1-28 (1911)

20. Schwab, Ch., Stevenson, R.: Space-time adaptive wavelet methods for parabolic evolution problems. Math. Comp. 78(267), 1293-1318 (2009) 